\title{
Efficient and Stable Numerical Methods for Multi-Term Time Fractional Sub-Diffusion Equations
}

\author{
Jincheng Ren ${ }^{1,2}$ and Zhi-zhong Sun ${ }^{2, *}$ \\ ${ }^{1}$ College of Mathematics and Information Science, Henan University of Economics \\ and Law, Zhengzhou 450000, China. \\ 2 Department of Mathematics, Southeast University, Nanjing 210096, China.
}

Received 18 November 2013; Accepted (in revised version) 28 May 2014

Available online 11 July 2014

\begin{abstract}
Some efficient numerical schemes are proposed for solving one-dimensional (1D) and two-dimensional (2D) multi-term time fractional sub-diffusion equations, combining the compact difference approach for the spatial discretisation and $L 1$ approximation for the multi-term time Caputo fractional derivatives. The stability and convergence of these difference schemes are theoretically established. Several numerical examples are implemented, testifying to their efficiency and confirming their convergence order.
\end{abstract}

AMS subject classifications: 65M06, 65M12, 65M15

Key words: Multi-term time fractional sub-diffusion equations, compact /compact ADI difference scheme, discrete energy method, convergence.

\section{Introduction}

Fractional differential equations are now frequently invoked in various scientific and engineering applications. Physical phenomena in fields such as viscoelasticity, diffusion processes, relaxation vibrations and electrochemistry are successfully described by differential equations involving derivatives of fractional order [1-8]. Moreover, some underlying processes that cannot be described by single term time fractional partial differential equations can be described by multi-term equations - e.g. the multi-term time fractional diffusion-wave equation modelling various types of viscoelastic damping [9].

In this article, we provide some numerical difference schemes to solve multi-term time fractional sub-diffusion equations of the following form [9-11]:

$$
P\left({ }^{C} D_{t}\right) u(x, t)=\kappa \frac{\partial^{2} u(x, t)}{\partial x^{2}}+f(x, t), \quad 0<x<L, 0<t \leq T
$$

${ }^{*}$ Corresponding author. Email addresses: renjincheng2001@126.com (J. Ren), zzsun@seu.edu.cn (Z. Sun) 
where $\kappa$ is a positive diffusion constant and the multi-term fractional operator $P\left({ }^{C} D_{t}\right)$ here is defined by

$$
P\left({ }^{C} D_{t}\right) v(x, t)=\left({ }_{0}^{C} D_{t}^{\alpha}+\sum_{i=1}^{s} a_{i}{ }_{0}^{C} D_{t}^{\alpha_{i}}\right) v(x, t)
$$

with $0<\alpha_{s}<\cdots<\alpha_{1}<\alpha<1$ and $a_{i} \in R, i=1,2, \cdots, s$, and ${ }_{0}^{C} D_{t}^{\alpha}$ denotes the Caputo fractional derivative of order $\alpha$ :

$$
{ }_{0}^{C} D_{t}^{\alpha} v(t)=\frac{1}{\Gamma(1-\alpha)} \int_{0}^{t} \frac{v^{\prime}(s)}{(t-s)^{\alpha}} d s .
$$

We note that Luchko [10] obtained a priori solution estimates for the generalised multiterm time fractional diffusion equation using an appropriate maximum principle, and established solution uniqueness by the Fourier method. Daftardar-Gejji \& Bhalekar [11] used separation of variables to solve a multi-term fractional diffusion-wave equation with homogeneous/nonhomogeneous boundary conditions. From Luchko's Theorem and the equivalent relationship between the Laplacian operator and the Riesz fractional derivative, Jiang et al. $[12,13]$ derived analytical solutions for both multi-term time-space fractional advection-diffusion and multi-term modified power law wave equations, respectively.

Other authors have discussed the numerical solution of fractional partial differential equations, including the fractional anomalous diffusion equation [14-24]. Chen et al. [14] proved the stability and convergence of an implicit difference approximation scheme of the fractional sub-diffusion equation using Fourier analysis. Lynch et al. [15] studied the numerical properties of the partial differential equations of fractional order $1<\alpha<2$. Yuste $[16,17]$ presented an explicit scheme and weighted average finite difference methods for the time fractional diffusion equation, and analysed their stability by the vonNeumann method. Zhuang et al. [18] obtained an implicit numerical method to solve the sub-diffusion equation by integrating the original equation on both sides, and proved the stability and convergence of their scheme by the energy method. Zhang et al. [19] constructed a Crank-Nicolson-type difference scheme and a compact difference scheme, to solve the time fractional sub-diffusion equation with a Riemann-Liouville fractional derivative. They proved that these two difference schemes are unconditionally stable, and the numerical solution converges in the maximum norm. Zhao \& Sun [20] provided a boxtype scheme for solving a class of fractional sub-diffusion equation with Neumann boundary conditions. Later, Ren et al. [21] proposed a compact difference scheme for the time fractional sub-diffusion equation with Neumann boundary conditions, and proved its unconditional stability and global convergence to be $O\left(\tau^{2-\alpha}+h^{4}\right)$ in the discrete $L_{2}$ norm.

There has also been some previous work on the numerical solution of problems with multiple fractional derivatives. Ford et al. [25] introduced a numerical method for solving the space-time fractional telegraph equation. Based on a quadrature formula approximation of the Caputo fractional derivative in spatial and temporal direction respectively, they proved the scheme was conditionally stable using the Fourier method. Liu et al. [26] proposed an implicit difference scheme for modified anomalous sub-diffusion equations with a nonlinear source term, and showed its convergence is $O\left(\tau+h^{2}\right)$ by the energy method. 
Recently, Liu et al. [28] discussed some computationally effective numerical methods for the multi-term time fractional wave-diffusion equations, and Yang et al. [29] applied the variational iteration method to obtain approximate solutions for multi-term fractional differential equations.

Since fractional derivatives are non-local operators, any low-order finite difference or finite element scheme requires a large number of operations and a large memory storage capacity, so it is very desirable to use high-order methods for the efficient numerical solution of fractional derivative problems. However, there appear to be very few previous studies on efficient numerical methods and their relevant stability and convergence analysis, for problems involving multi-term fractional partial differential equations. The main purpose of this article is to construct effective and fast numerical methods for the $1 \mathrm{D}$ and $2 \mathrm{D}$ forms of the multi-term time fractional sub-diffusion equation (1.1), and to establish corresponding error estimates. To reduce the computational burden, we adopt fourth-order compact differences for the spatial approximation $[19,21,33]$, such that fewer grid points are required to produce accurate solutions. The $L 1$ approximation proposed by $\mathrm{Xu}$ [30] and Sun [31] is adopted to deal with the multi-term temporal Caputo fractional derivatives. Using the discrete energy method, we prove that our resulting compact difference scheme is unconditionally stable and globally convergent, with the convergence $O\left(\tau^{2-\alpha}+h^{4}\right)$ in the maximum norm. Furthermore, another new scheme with secondorder spatial accuracy is also presented, and its corresponding stability and convergence discussed.

In Section 2, we first give some auxiliary lemmas, and then derive our compact difference scheme. In Section 3, by introducing a new inner product and using the energy method, we prove the stability and convergence of the compact difference scheme. In Section 4, the second-order scheme with unconditional stability and maximum norm convergence is discussed. Some results on the two-dimensional multi-term time fractional diffusion equation are given in Sections 5 and 6, respectively. In Section 7, some numerical experiments are presented to support the theoretical analysis, and to show the efficiency of the difference scheme. Some final comments are made in the concluding section.

\section{Construction of the Compact Finite Difference Scheme}

\subsection{Notation and auxiliary lemmas}

We first give some notation and auxiliary lemmas, to be used in the construction of the compact finite difference scheme. Without loss of generality, we may take $a_{1}=1$ and $\kappa=1$ in Eq. (1.1) and consider the following problem involving the 1D two-term time fractional sub-diffusion equation:

$$
\begin{aligned}
& { }_{0}^{C} D_{t}^{\alpha_{1}} u(x, t)+{ }_{0}^{C} D_{t}^{\alpha} u(x, t)=\frac{\partial^{2} u(x, t)}{\partial x^{2}}+f(x, t), 0<x<L, 0<t \leq T, \\
& u(x, 0)=\varphi(x), \quad 0 \leq x \leq L, \\
& u(0, t)=\psi_{1}(t), u(L, t)=\psi_{2}(t), 0<t \leq T,
\end{aligned}
$$


where $0<\alpha_{1}<\alpha<1$ and $\varphi(x), \psi_{1}(t), \psi_{2}(t)$ and $f(x, t)$ are known smooth functions.

For the finite difference approximation, we set $h=L / M$ and $\tau=T / N$, where $M$ and $N$ are two positive integers. The domain $[0, L] \times[0, T]$ is covered by $\Omega_{h} \times \Omega_{\tau}$, where $\Omega_{h}=\left\{x_{i} \mid x_{i}=i h, 0 \leq i \leq M\right\}$ and $\Omega_{\tau}=\left\{t_{n} \mid t_{n}=n \tau, 0 \leq n \leq N\right\}$. For any grid function $u=\left\{u_{j}^{k} \mid 0 \leq j \leq M, 0 \leq k \leq N\right\}$ defined on $\Omega_{h} \times \Omega_{\tau}$, we introduce the notation

$$
\delta_{x} u_{j-\frac{1}{2}}^{k}=\frac{1}{h}\left(u_{j}^{k}-u_{j-1}^{k}\right), \quad \delta_{x}^{2} u_{j}^{k}=\frac{1}{h}\left(\delta_{x} u_{j+\frac{1}{2}}^{k}-\delta_{x} u_{j-\frac{1}{2}}^{k}\right) .
$$

For convenience, we denote a discrete fractional derivative operator $D_{\tau}^{\alpha}$ and an average operator $A$ respectively as follows:

$$
D_{\tau}^{\alpha} u_{i}^{k}=\frac{1}{\mu}\left[u_{i}^{k}-\sum_{j=1}^{k-1}\left(a_{k-j-1}^{\alpha}-a_{k-j}^{\alpha}\right) u_{i}^{j}-a_{k-1}^{\alpha} \varphi\left(x_{i}\right)\right], 0 \leq i \leq M, \quad 1 \leq k \leq N,
$$

where $\mu=\tau^{\alpha} \Gamma(2-\alpha)$ and $a_{k}^{\alpha}=(k+1)^{1-\alpha}-k^{1-\alpha}$; and

$$
A u_{i}^{k}=\left\{\begin{array}{l}
\frac{1}{12}\left(u_{i-1}^{k}+10 u_{i}^{k}+u_{i+1}^{k}\right)=\left(I+\frac{h^{2}}{12} \delta_{x}^{2}\right) u_{i}^{k}, \quad 1 \leq i \leq M-1, \quad 0 \leq k \leq N, \\
u_{i}^{k}, \quad i=0, M, \quad 0 \leq k \leq N,
\end{array}\right.
$$

where $I$ denotes the identity operator.

The following lemmas will be used in deriving the difference schemes.

Lemma 2.1 (cf. Ref. [31]). Suppose that $0<\alpha<1, g \in C^{2}\left[0, t_{n}\right]$, and

$$
\begin{aligned}
R^{\alpha}\left(g\left(t_{n}\right)\right) \equiv & \frac{1}{\Gamma(1-\alpha)} \int_{0}^{t_{n}} \frac{g^{\prime}(s)}{\left(t_{n}-s\right)^{\alpha}} d s \\
& -\frac{\tau^{-\alpha}}{\Gamma(2-\alpha)}\left[a_{0}^{\alpha} g\left(t_{n}\right)-\sum_{k=1}^{n-1}\left(a_{n-k-1}^{\alpha}-a_{n-k}^{\alpha}\right) g\left(t_{k}\right)-a_{n-1}^{\alpha} g(0)\right] .
\end{aligned}
$$

Then

$$
\left|R^{\alpha}\left(g\left(t_{n}\right)\right)\right| \leq \frac{1}{\Gamma(2-\alpha)}\left[\frac{1-\alpha}{12}+\frac{2^{2-\alpha}}{2-\alpha}-\left(1+2^{-\alpha}\right)\right] \max _{0 \leq t \leq t_{n}}\left|g^{\prime \prime}(t)\right| \tau^{2-\alpha} .
$$

Lemma 2.2 (cf. Ref [32]). Denote $\zeta(s)=(1-s)^{3}\left[5-3(1-s)^{2}\right]$. Then if $f(x) \in C^{6}\left[x_{i-1}, x_{i+1}\right]$ where $1 \leq i \leq M-1$,

$$
\begin{aligned}
& \frac{1}{12}\left[f^{\prime \prime}\left(x_{i-1}\right)+10 f^{\prime \prime}\left(x_{i}\right)+f^{\prime \prime}\left(x_{i+1}\right)\right]-\frac{1}{h^{2}}\left[f\left(x_{i-1}\right)-2 f\left(x_{i}\right)+f\left(x_{i+1}\right)\right] \\
= & \frac{h^{4}}{360} \int_{0}^{1}\left[f^{(6)}\left(x_{i}-s h\right)+f^{(6)}\left(x_{i}+s h\right)\right] \zeta(s) d s .
\end{aligned}
$$




\subsection{Derivation of the compact difference scheme}

Let us now construct a compact difference scheme to solve the problem (2.1)-(2.3). On $\Omega_{h} \times \Omega_{\tau}$, we define the grid functions

$$
U_{i}^{k}=u\left(x_{i}, t_{k}\right), \quad f_{i}^{k}=f\left(x_{i}, t_{k}\right), \quad 0 \leq i \leq M, \quad 0 \leq k \leq N .
$$

Suppose $u(x, t) \in C_{x, t}^{6,2}([0, L] \times[0, T])$. Considering Eq. (2.1) at the point $\left(x_{i}, t_{k}\right)$, we have

$$
{ }_{0}^{C} D_{t}^{\alpha_{1}} u\left(x_{i}, t_{k}\right)+{ }_{0}^{C} D_{t}^{\alpha} u\left(x_{i}, t_{k}\right)=\frac{\partial^{2} u\left(x_{i}, t_{k}\right)}{\partial x^{2}}+f\left(x_{i}, t_{k}\right), \quad 0 \leq i \leq M, 1 \leq k \leq N,
$$

and on applying the average operator $A$ to both sides:

$$
A_{0}^{C} D_{t}^{\alpha_{1}} u\left(x_{i}, t_{k}\right)+A_{0}^{C} D_{t}^{\alpha} u\left(x_{i}, t_{k}\right)=A \frac{\partial^{2} u\left(x_{i}, t_{k}\right)}{\partial x^{2}}+A f\left(x_{i}, t_{k}\right), 1 \leq i \leq M-1,1 \leq k \leq N .
$$

From Lemmas 2.1 and 2.2 and recalling $D_{\tau}^{\alpha_{1}}$ and $D_{\tau}^{\alpha}$,

$$
A D_{\tau}^{\alpha_{1}} U_{i}^{k}+A D_{\tau}^{\alpha} U_{i}^{k}=\delta_{x}^{2} U_{i}^{k}+A f_{i}^{k}+R_{i}^{k}, \quad 1 \leq i \leq M-1,1 \leq k \leq N,
$$

where

$$
\begin{aligned}
R_{i}^{k}= & \frac{h^{4}}{360} \int_{0}^{1}\left[\frac{\partial^{6} u}{\partial x^{6}}\left(x_{i}-s h, t_{k}\right)+\frac{\partial^{6} u}{\partial x^{6}}\left(x_{i}+s h, t_{k}\right)\right] \zeta(s) d s \\
& +A R^{\alpha_{1}}\left(u\left(x_{i}, t_{k}\right)\right)+A R^{\alpha}\left(u\left(x_{i}, t_{k}\right)\right) .
\end{aligned}
$$

Noting that $0<\alpha_{1}<\alpha<1$, there exists a positive constant $c_{1}$ independent of $h$ and $\tau$ such that

$$
\left|R_{i}^{k}\right| \leq c_{1}\left(\tau^{2-\alpha}+h^{4}\right), \quad 1 \leq i \leq M-1, \quad 1 \leq k \leq N .
$$

In addition, it follows from the initial and boundary conditions (2.2) and (2.3) that

$$
\begin{array}{ll}
U_{i}^{0}=\varphi\left(x_{i}\right), & 0 \leq i \leq M, \\
U_{0}^{k}=\psi_{1}\left(t_{k}\right), & U_{M}^{k}=\psi_{2}\left(t_{k}\right), \quad 1 \leq k \leq N .
\end{array}
$$

Omitting the small term $R_{i}^{k}$ in Eq. (2.4) and replacing the function $U_{i}^{k}$ with its numerical approximation $u_{i}^{k}$, on noting (2.6) and (2.7) we construct the following compact difference scheme (L1-CD):

$$
\begin{aligned}
& A D_{\tau}^{\alpha_{1}} u_{i}^{k}+A D_{\tau}^{\alpha} u_{i}^{k}=\delta_{x}^{2} u_{i}^{k}+A f_{i}^{k}, \quad 1 \leq i \leq M-1,1 \leq k \leq N, \\
& u_{i}^{0}=\varphi\left(x_{i}\right), \quad 0 \leq i \leq M, \\
& u_{0}^{k}=\psi_{1}\left(t_{k}\right), \quad u_{M}^{k}=\psi_{2}\left(t_{k}\right), \quad 1 \leq k \leq N .
\end{aligned}
$$

It is easy to see that at each time level, this L1-CD scheme (2.8)-(2.10) is a linear tridiagonal system with a strictly diagonally dominant coefficient matrix, so we have the following theorem:

Theorem 2.1. The L1-CD scheme (2.8)-(2.10) is uniquely solvable. 


\section{Stability and Convergence of the L1-CD Scheme}

We now proceed to investigate of the stability and convergence of the L1-CD scheme (2.8)-(2.10). Let $V_{h}=\left\{v \mid v=\left(v_{0}, v_{1}, \cdots, v_{M-1}, v_{M}\right), v_{0}=v_{M}=0\right\}$. For any $u, v \in V_{h}$, we define the discrete inner product, $L_{2}$ norm, $H^{1}$ semi-norm and maximum norm as follows:

$$
\begin{aligned}
& (u, v)=h \sum_{i=1}^{M-1} u_{i} v_{i}, \quad\|u\|=\sqrt{(u, u)}, \\
& \left\langle\delta_{x} u, \delta_{x} v\right\rangle=h \sum_{i=0}^{M-1}\left(\delta_{x} u_{i+\frac{1}{2}}\right)\left(\delta_{x} v_{i+\frac{1}{2}}\right)-\frac{h^{3}}{12} \sum_{i=1}^{M-1}\left(\delta_{x}^{2} u_{i}\right)\left(\delta_{x}^{2} v_{i}\right), \quad\left\|\delta_{x} u\right\|_{h}=\sqrt{\left\langle\delta_{x} u, \delta_{x} u\right\rangle}, \\
& |u|_{1}=\sqrt{h \sum_{i=0}^{M-1}\left(\delta_{x} u_{i+\frac{1}{2}}\right)^{2}}, \quad\|u\|_{\infty}=\max _{1 \leq i \leq M-1}\left|u_{i}\right| .
\end{aligned}
$$

Lemma 3.1 (cf. Refs. [14, 19]). If $0<\gamma<1$ and $a_{k}^{\gamma}=(k+1)^{1-\gamma}-k^{1-\gamma}, k=0,1,2, \cdots$. Then

$$
\begin{aligned}
& 1=a_{0}^{\gamma}>a_{1}^{\gamma}>a_{2}^{\gamma}>\cdots>a_{k}^{\gamma}>\cdots \rightarrow 0, \\
& (1-\gamma)(k+1)^{-\gamma}<a_{k}^{\gamma}<(1-\gamma) k^{-\gamma} .
\end{aligned}
$$

Lemma 3.2. Let $0<\gamma<1$. For any grid function $u=\left\{u_{i}^{k} \mid 0 \leq i \leq M, 0 \leq k \leq N\right\}$ defined on $\Omega_{h} \times \Omega_{\tau}$, it holds that

$$
-\left(A D_{\tau}^{\gamma} u^{k}, \delta_{x}^{2} u^{k}\right)=\frac{1}{\mu}\left[\left\|\delta_{x} u^{k}\right\|_{h}^{2}-\sum_{j=1}^{k-1}\left(a_{k-j-1}^{\gamma}-a_{k-j}^{\gamma}\right)\left\langle\delta_{x} u^{j}, \delta_{x} u^{k}\right\rangle-a_{k-1}^{\gamma}\left\langle\delta_{x} u^{0}, \delta_{x} u^{k}\right\rangle\right],
$$

where $\mu=\tau^{\gamma} \Gamma(2-\gamma)$.

Proof. From the definition of $D_{\tau}^{\gamma}$ and the discrete Green formula,

$$
\begin{aligned}
& -\mu\left(A D_{\tau}^{\gamma} u^{k}, \delta_{x}^{2} u^{k}\right) \\
= & -\left(\left(I+\frac{h^{2}}{12} \delta_{x}^{2}\right) u^{k}, \delta_{x}^{2} u^{k}\right)+\sum_{j=1}^{k-1}\left(a_{k-j-1}^{\gamma}-a_{k-j}^{\gamma}\right)\left(\left(I+\frac{h^{2}}{12} \delta_{x}^{2}\right) u^{j}, \delta_{x}^{2} u^{k}\right) \\
& +a_{k-1}^{\gamma}\left(\left(I+\frac{h^{2}}{12} \delta_{x}^{2}\right) u^{0}, \delta_{x}^{2} u^{k}\right) \\
= & \left(\left\|\delta_{x} u^{k}\right\|^{2}-\frac{h^{2}}{12}\left\|\delta_{x}^{2} u^{k}\right\|^{2}\right)-\sum_{j=1}^{k-1}\left(a_{k-j-1}^{\gamma}-a_{k-j}^{\gamma}\right)\left[\left(\delta_{x} u^{j}, \delta_{x} u^{k}\right)-\frac{h^{2}}{12}\left(\delta_{x}^{2} u^{j}, \delta_{x}^{2} u^{k}\right)\right] \\
& -a_{k-1}^{\gamma}\left[\left(\delta_{x} u^{0}, \delta_{x} u^{k}\right)-\frac{h^{2}}{12}\left(\delta_{x}^{2} u^{0}, \delta_{x}^{2} u^{k}\right)\right] \\
= & \left\|\delta_{x} u^{k}\right\|_{h}^{2}-\sum_{j=1}^{k-1}\left(a_{k-j-1}^{\gamma}-a_{k-j}^{\gamma}\right)\left\langle\delta_{x} u^{j}, \delta_{x} u^{k}\right\rangle-a_{k-1}^{\gamma}\left\langle\delta_{x} u^{0}, \delta_{x} u^{k}\right\rangle .
\end{aligned}
$$


Dividing the above equality by $\mu$ then yields the desired result.

Lemma 3.3 (cf. Ref. [33]). For any mesh function $u \in V_{h}$,

$$
\frac{2}{3}|u|_{1}^{2} \leq\left\|\delta_{x} u\right\|_{h}^{2} \leq|u|_{1}^{2} .
$$

The semi-norm $\|\cdot\|_{h}$ is equivalent to the standard $H^{1}$ semi-norm, but the semi-norm $\|\cdot\|_{h}$ is more convenient than the standard $H^{1}$ semi-norm for the stability and convergence analysis. We now prove the following Theorem on an a priori estimate.

Theorem 3.1. Let $\left\{v_{i}^{k} \mid 0 \leq i \leq M, 0 \leq k \leq N\right\}$ be the solution of the difference system

$$
\begin{aligned}
& A D_{\tau}^{\alpha_{1}} v_{i}^{k}+A D_{\tau}^{\alpha} v_{i}^{k}=\delta_{x}^{2} v_{i}^{k}+g_{i}^{k}, \quad 1 \leq i \leq M-1,1 \leq k \leq N, \\
& v_{i}^{0}=v^{0}\left(x_{i}\right), \quad 0 \leq i \leq M, \\
& v_{0}^{k}=0, \quad v_{M}^{k}=0, \quad 1 \leq k \leq N,
\end{aligned}
$$

where $v^{0}\left(x_{0}\right)=v^{0}\left(x_{M}\right)=0$. Then

$$
\left\|\delta_{x} v^{k}\right\|_{h}^{2} \leq\left\|\delta_{x} v^{0}\right\|_{h}^{2}+\frac{c_{\max }}{4} \max _{1 \leq l \leq N}\left\|g^{l}\right\|^{2},
$$

where $c_{\max }=\max \left\{T^{\alpha_{1}} \Gamma\left(1-\alpha_{1}\right), T^{\alpha} \Gamma(1-\alpha)\right\}$.

Proof. Taking the inner product of Eq. (3.1) with $-\delta_{x}^{2} v^{k}$, we have

$$
-\left(A D_{\tau}^{\alpha_{1}} v^{k}, \delta_{x}^{2} \nu^{k}\right)-\left(A D_{\tau}^{\alpha} v^{k}, \delta_{x}^{2} \nu^{k}\right)=-\left(\delta_{x}^{2} \nu^{k}, \delta_{x}^{2} \nu^{k}\right)-\left(g^{k}, \delta_{x}^{2} \nu^{k}\right), \quad 1 \leq k \leq N ;
$$

and hence from Lemma 3.2

$$
\begin{aligned}
-\left(A D_{\tau}^{\alpha_{1}} v^{k}, \delta_{x}^{2} v^{k}\right)= & \frac{1}{\mu_{1}}\left\|\delta_{x} v^{k}\right\|_{h}^{2}-\frac{1}{\mu_{1}} \sum_{j=1}^{k-1}\left(a_{k-j-1}^{\alpha_{1}}-a_{k-j}^{\alpha_{1}}\right)\left\langle\delta_{x} v^{j}, \delta_{x} v^{k}\right\rangle \\
& -\frac{a_{k-1}^{\alpha_{1}}}{\mu_{1}}\left\langle\delta_{x} v^{0}, \delta_{x} v^{k}\right\rangle, \\
-\left(A D_{\tau}^{\alpha} v^{k}, \delta_{x}^{2} v^{k}\right)= & \frac{1}{\mu_{0}}\left\|\delta_{x} v^{k}\right\|_{h}^{2}-\frac{1}{\mu_{0}} \sum_{j=1}^{k-1}\left(a_{k-j-1}^{\alpha}-a_{k-j}^{\alpha}\right)\left\langle\delta_{x} v^{j}, \delta_{x} v^{k}\right\rangle \\
& -\frac{a_{k-1}^{\alpha}}{\mu_{0}}\left\langle\delta_{x} v^{0}, \delta_{x} v^{k}\right\rangle .
\end{aligned}
$$

Substituting Eqs. (3.5) and (3.6) into Eq. (3.4), from Lemma 3.1 and the Cauchy-Schwarz inequality we consequently deduce that

$$
\begin{aligned}
& \left(\frac{1}{\mu_{1}}+\frac{1}{\mu_{0}}\right)\left\|\delta_{x} v^{k}\right\|_{h}^{2} \\
\leq & \sum_{j=1}^{k-1}\left[\frac{1}{\mu_{1}}\left(a_{k-j-1}^{\alpha_{1}}-a_{k-j}^{\alpha_{1}}\right)+\frac{1}{\mu_{0}}\left(a_{k-j-1}^{\alpha}-a_{k-j}^{\alpha}\right)\right]\left\|\delta_{x} v^{j}\right\|_{h}^{2} \\
& +\frac{a_{k-1}^{\alpha_{1}}}{\mu_{1}}\left(\left\|\delta_{x} v^{0}\right\|_{h}^{2}+\frac{\mu_{1}}{4 a_{k-1}^{\alpha_{1}}}\left\|g^{k}\right\|^{2}\right)+\frac{a_{k-1}^{\alpha}}{\mu_{0}}\left(\left\|\delta_{x} v^{0}\right\|_{h}^{2}+\frac{\mu_{0}}{4 a_{k-1}^{\alpha}}\left\|g^{k}\right\|^{2}\right) .
\end{aligned}
$$


Applying Lemma 3.1 and noting that $0<\alpha_{1}<\alpha<1$, we also obtain

$$
\begin{aligned}
& \frac{\mu_{1}}{4 a_{k-1}^{\alpha_{1}}}<\frac{k^{\alpha_{1}} \tau^{\alpha_{1}} \Gamma\left(2-\alpha_{1}\right)}{4\left(1-\alpha_{1}\right)} \leq \frac{T^{\alpha_{1}} \Gamma\left(1-\alpha_{1}\right)}{4}, \\
& \frac{\mu_{0}}{4 a_{k-1}^{\alpha}}<\frac{k^{\alpha} \tau^{\alpha} \Gamma(2-\alpha)}{4(1-\alpha)} \leq \frac{T^{\alpha} \Gamma(1-\alpha)}{4} .
\end{aligned}
$$

Letting

$$
B=\left\|\delta_{x} v^{0}\right\|_{h}^{2}+\frac{c_{\max }}{4}\left\|g^{l}\right\|^{2}
$$

and substituting the above estimates into Eq. (3.7), we have

$$
\begin{aligned}
\left(\frac{1}{\mu_{1}}+\frac{1}{\mu_{0}}\right)\left\|\delta_{x} v^{k}\right\|_{h}^{2} \leq & \sum_{j=1}^{k-1}\left[\frac{1}{\mu_{1}}\left(a_{k-j-1}^{\alpha_{1}}-a_{k-j}^{\alpha_{1}}\right)+\frac{1}{\mu_{0}}\left(a_{k-j-1}^{\alpha}-a_{k-j}^{\alpha}\right)\right]\left\|\delta_{x} v^{j}\right\|_{h}^{2} \\
& +\left(\frac{a_{k-1}^{\alpha_{1}}}{\mu_{1}}+\frac{a_{k-1}^{\alpha}}{\mu_{0}}\right) B, \quad 1 \leq k \leq N .
\end{aligned}
$$

Using the same arguments as in $[20,21]$, we obtain the desired result.

We obtain the following stability statement from Theorem 3.1.

Theorem 3.2. The L1-CD scheme (2.8)-(2.10) is unconditionally stable to the initial value $\varphi$ and the inhomogeneous term $f$.

Let us now consider the convergence of the L1-CD scheme (2.8)-(2.10). Writing

$$
e_{i}^{k}=U_{i}^{k}-u_{i}^{k}, \quad 0 \leq i \leq M, \quad 0 \leq k \leq N,
$$

and subtracting Eqs. (2.8)-(2.10) from Eq. (2.4) and Eqs. (2.6)-(2.7) respectively, we get the error equations

$$
\begin{aligned}
& A D_{\tau}^{\alpha_{1}} e_{i}^{k}+A D_{\tau}^{\alpha} e_{i}^{k}=\delta_{x}^{2} e_{i}^{k}+R_{i}^{k}, \quad 1 \leq i \leq M-1,1 \leq k \leq N, \\
& e_{i}^{0}=0, \quad 0 \leq i \leq M, \\
& e_{0}^{k}=0, \quad e_{M}^{k}=0, \quad 1 \leq k \leq N .
\end{aligned}
$$

From Eq. (2.5) and Theorem 3.1 we have

$$
\left\|\delta_{x} e^{k}\right\|_{h}^{2} \leq \frac{c_{\max }}{4} \max _{1 \leq l \leq N}\left\|R^{l}\right\|^{2} \leq \frac{c_{\max } L c_{1}^{2}}{4}\left(\tau^{2-\alpha}+h^{4}\right)^{2},
$$

and then applying the embedding inequality $\|u\|_{\infty} \leq \frac{\sqrt{L}}{2}|u|_{1}$ (cf. [34]) and Lemma 3.3 we obtain the following convergence result.

Theorem 3.3. Assume that $u(x, t) \in C_{x, t}^{6,2}([0, L] \times[0, T])$ is the solution of Eqs. (2.1)-(2.3) and $\left\{u_{i}^{k} \mid 0 \leq i \leq M, 0 \leq k \leq N\right\}$ is the solution of the L1-CD scheme Eqs. (2.8)-(2.10). Then

$$
\left\|e^{k}\right\|_{\infty} \leq \frac{c_{1} L}{8} \sqrt{6 c_{\max }}\left(\tau^{2-\alpha}+h^{4}\right), \quad 0 \leq k \leq N .
$$




\section{A Second-Order Finite Difference Scheme}

We now present a finite difference scheme second-order in the spatial direction that is computationally efficient when the storage is inexpensive. Thus for the problem (2.1)(2.3) we construct the L1-SOD scheme

$$
\begin{aligned}
& D_{\tau}^{\alpha_{1}} u_{i}^{k}+D_{\tau}^{\alpha} u_{i}^{k}=\delta_{x}^{2} u_{i}^{k}+f_{i}^{k}, \quad 1 \leq i \leq M-1,1 \leq k \leq N \\
& u_{i}^{0}=\varphi\left(x_{i}\right), \quad 0 \leq i \leq M \\
& u_{0}^{k}=\psi_{1}\left(t_{k}\right), \quad u_{M}^{k}=\psi_{2}\left(t_{k}\right), \quad 1 \leq k \leq N
\end{aligned}
$$

Lemma 4.1. Suppose $u(x, t) \in C_{x, t}^{4,2}([0, L] \times[0, T])$ is the solution of the problem (2.1)(2.3). Then the truncation error of the L1-SOD scheme (4.1)-(4.3) satisfies

$$
\left|\hat{R}_{i}^{k}\right| \leq \hat{c}_{R}\left(\tau^{2-\alpha}+h^{2}\right), \quad 0 \leq i \leq M, \quad 1 \leq k \leq N,
$$

where $\hat{c}_{R}$ is a positive constant independent of $\tau$ and $h$.

Using the analytical method of the compact difference scheme in Section 3, we can also prove that the L1-SOD scheme (4.1)-(4.3) is stable to the initial value $\varphi$ and the forcing term $f$, and convergent with the convergence order of $O\left(\tau^{2-\alpha}+h^{2}\right)$ in the maximum norm.

Theorem 4.1. Suppose $\left\{u_{i}^{k} \mid 0 \leq i \leq M, 0 \leq k \leq N\right\}$ is the solution of the L1-SOD scheme (4.1)-(4.3). Then

$$
\left\|\delta_{x} u^{k}\right\|^{2} \leq\left\|\delta_{x} u^{0}\right\|^{2}+\frac{c_{\max }}{4} \max _{1 \leq l \leq N}\left\|f^{l}\right\|^{2}
$$

Theorem 4.2. If the problem (2.1)-(2.3) has smooth solution $u(x, t) \in C_{x, t}^{4,2}([0, L] \times[0, T])$ and $\left\{u_{i}^{k} \mid 0 \leq i \leq M, 0 \leq k \leq N\right\}$ is the solution of the L1-SOD scheme (4.1)-(4.3), then

$$
\left\|U^{k}-u^{k}\right\|_{\infty} \leq \frac{\hat{c}_{R} L}{4} \sqrt{c_{\max }}\left(\tau^{2-\alpha}+h^{2}\right), \quad 0 \leq k \leq N .
$$

\section{Extension to 2D Multi-Term Time-Fractional Sub-Diffusion Equations}

Let is now consider the numerical solution of the following problem involving the $2 \mathrm{D}$ multi-term time fractional sub-diffusion equations:

$$
\begin{aligned}
& { }_{0}^{C} D_{t}^{\alpha_{1}} u(x, y, t)+{ }_{0}^{C} D_{t}^{\alpha} u(x, y, t)=\Delta u(x, y, t)+f(x, y, t), \quad(x, y) \in \Omega, 0<t \leq T, \\
& u(x, y, 0)=\varphi(x, y), \quad(x, y) \in \bar{\Omega}=\Omega \cup \partial \Omega, \\
& u(x, y, t)=\psi(x, y, t), \quad(x, y) \in \partial \Omega, 0<t \leq T,
\end{aligned}
$$

where $\Omega=\left(0, L_{1}\right) \times\left(0, L_{2}\right), \partial \Omega$ is the boundary, $0<\alpha_{1}<\alpha<1$, and $\varphi(x, y), \psi(x, y, t)$ and $f(x, y, t)$ are known smooth functions. 
Set $x_{i}=i h_{1}$ and $y_{j}=j h_{2}$ with $h_{1}=L_{1} / M_{1}$ and $h_{2}=L_{2} / M_{2}$, where $M_{1}$ and $M_{2}$ are positive integers. Define $\Omega_{h_{1}}=\left\{x_{i} \mid 0 \leq i \leq M_{1}\right\}$ and $\Omega_{h_{2}}=\left\{y_{j} \mid 0 \leq j \leq M_{2}\right\}$, $\bar{\Omega}_{h}=\Omega_{h_{1}} \times \Omega_{h_{2}}, \Omega_{h}=\bar{\Omega}_{h} \cup \Omega$ and $\partial \Omega_{h}=\bar{\Omega}_{h} \cup \partial \Omega$. The definitions of $\tau, t_{k}$ are the same in Section 2. For any grid function $v=\left\{v_{i j} \mid 0 \leq i \leq M_{1}, 0 \leq j \leq M_{2}\right\}$, we denote

$$
\begin{array}{ll}
\delta_{x} v_{i-\frac{1}{2}, j}=\frac{1}{h_{1}}\left(v_{i j}-v_{i-1, j}\right), & \delta_{x}^{2} v_{i j}=\frac{1}{h_{1}}\left(\delta_{x} v_{i+\frac{1}{2}, j}-\delta_{x} v_{i-\frac{1}{2}, j}\right), \\
\delta_{y} \delta_{x} v_{i-\frac{1}{2}, j-\frac{1}{2}}=\frac{1}{h_{2}}\left(\delta_{x} v_{i-\frac{1}{2}, j}-\delta_{x} v_{i-\frac{1}{2}, j-1}\right), & \delta_{y} \delta_{x}^{2} v_{i, j-\frac{1}{2}}=\frac{1}{h_{2}}\left(\delta_{x}^{2} v_{i j}-\delta_{x}^{2} v_{i, j-1}\right) .
\end{array}
$$

Similarly, the notations $\delta_{y} v_{i, j-\frac{1}{2}}, \delta_{y}^{2} v_{i j}, \delta_{x} \delta_{y}^{2} v_{i-\frac{1}{2}, j}, \delta_{x}^{2} \delta_{y}^{2} v_{i j}$ can be defined. The discrete Laplace operator is denoted $\Delta_{h} v_{i j}=\delta_{x}^{2} v_{i j}+\delta_{y}^{2} v_{i j}$, and the spatial average difference operators are defined as

$$
\begin{aligned}
& H_{x} v_{i j}= \begin{cases}\frac{1}{12}\left(v_{i-1, j}+10 v_{i j}+v_{i+1, j}\right)=\left(I+\frac{h_{1}^{2}}{12} \delta_{x}^{2}\right) v_{i j}, & 1 \leq i \leq M_{1}-1,0 \leq j \leq M_{2}, \\
v_{i j}, & i=0 \text { or } M_{1}, 0 \leq j \leq M_{2},\end{cases} \\
& H_{y} v_{i j}= \begin{cases}\frac{1}{12}\left(v_{i, j-1}+10 v_{i j}+v_{i, j+1}\right)=\left(I+\frac{h_{2}^{2}}{12} \delta_{y}^{2}\right) v_{i j}, & 1 \leq j \leq M_{2}-1,0 \leq i \leq M_{1}, \\
v_{i j}, & j=0 \text { or } M_{2}, 0 \leq i \leq M_{1} .\end{cases}
\end{aligned}
$$

We introduce the space of grid functions on $\bar{\Omega}_{h}$ :

$$
V_{h}^{*}=\left\{v \mid v=\left\{v_{i j} \mid\left(x_{i}, y_{j}\right) \in \bar{\Omega}_{h}\right\} \text { and } v_{i j}=0 \text { if }\left(x_{i}, y_{j}\right) \in \partial \Omega_{h}\right\} .
$$

For any grid functions $u, v \in V_{h}^{*}$, we define the discrete inner product

$$
(u, v)=h_{1} h_{2} \sum_{i=1}^{M_{1}-1} \sum_{j=1}^{M_{2}-1} u_{i j} v_{i j}
$$

and denote $\|v\|=\sqrt{(v, v)}$. Similarly, we define $\left\|\delta_{x}^{2} v\right\|,\left\|\delta_{y}^{2} v\right\|$ and $\left\|\delta_{x}^{2} \delta_{y}^{2} v\right\|$; and denote

$$
\begin{aligned}
& \left\|\delta_{x} v\right\|=\sqrt{h_{1} h_{2} \sum_{i=1}^{M_{1}} \sum_{j=1}^{M_{2}-1}\left|\delta_{x} v_{i-\frac{1}{2}, j}\right|^{2}}, \\
& \left\|\delta_{x} \delta_{y} v\right\|=\sqrt{h_{1} h_{2} \sum_{i=1}^{M_{1}} \sum_{j=1}^{M_{2}}\left|\delta_{x} \delta_{y} v_{i-\frac{1}{2}, j-\frac{1}{2}}\right|^{2}} \\
& \left\|\delta_{y} \delta_{x}^{2} v\right\|=\sqrt{h_{1} h_{2} \sum_{i=1}^{M_{1}-1} \sum_{j=1}^{M_{2}}\left|\delta_{y} \delta_{x}^{2} v_{i j-\frac{1}{2}}\right|^{2}}
\end{aligned}
$$


and $\left\|\delta_{y} v\right\|,\left\|\delta_{x} \delta_{y}^{2} v\right\|$ similarly. The discrete $H^{1}$ semi-norm and $H^{1}$ norm are

$$
\left\|\nabla_{h} v\right\|=\sqrt{\left\|\delta_{x} v\right\|^{2}+\left\|\delta_{y} v\right\|^{2}}, \quad\|v\|_{H^{1}}=\sqrt{\|v\|^{2}+\left\|\nabla_{h} v\right\|^{2}} .
$$

Finally, for any grid functions $u, v \in V_{h}^{*}$ we denote

$$
\begin{aligned}
& \left\langle\nabla_{h} u, \nabla_{h} v\right\rangle_{h^{*}} \\
= & \left(\delta_{x} u, \delta_{x} v\right)+\left(\delta_{y} u, \delta_{y} v\right)-\frac{h_{1}^{2}}{12}\left[\left(\delta_{x}^{2} u, \delta_{x}^{2} v\right)+\left(\delta_{x} \delta_{y} u, \delta_{x} \delta_{y} v\right)\right] \\
& -\frac{h_{2}^{2}}{12}\left[\left(\delta_{y}^{2} u, \delta_{y}^{2} v\right)+\left(\delta_{x} \delta_{y} u, \delta_{x} \delta_{y} v\right)\right]+\frac{h_{1}^{2} h_{2}^{2}}{144}\left[\left(\delta_{x}^{2} \delta_{y} u, \delta_{x}^{2} \delta_{y} v\right)+\left(\delta_{x} \delta_{y}^{2} u, \delta_{x} \delta_{y}^{2} v\right)\right]
\end{aligned}
$$

and

$$
\left\|\nabla_{h} u\right\|_{h^{*}}=\sqrt{\left\langle\nabla_{h} u, \nabla_{h} u\right\rangle_{h^{*}}} .
$$

\subsection{Derivation of the compact ADI difference scheme}

We suppose that $u(x, y, t) \in C_{x, y, t}^{6,6,2}(\Omega \times[0, T])$, and define the grid functions

$$
U_{i j}^{n}=u\left(x_{i}, y_{j}, t_{n}\right), f_{i j}^{n}=f\left(x_{i}, y_{j}, t_{n}\right), \quad\left(x_{i}, y_{j}\right) \in \Omega_{h}, \quad 1 \leq n \leq N .
$$

Considering Eq. (5.1) at the point $\left(x_{i}, y_{j}, t_{n}\right)$, we have

$$
\begin{array}{r}
{ }_{0}^{C} D_{t}^{\alpha_{1}} u\left(x_{i}, y_{j}, t_{n}\right)+{ }_{0}^{C} D_{t}^{\alpha} u\left(x_{i}, y_{j}, t_{n}\right)=\Delta u\left(x_{i}, y_{j}, t_{n}\right)+f\left(x_{i}, y_{j}, t_{n}\right), \\
\left(x_{i}, y_{j}\right) \in \Omega_{h}, \quad 1 \leq n \leq N .
\end{array}
$$

Applying the average operator $H_{x} H_{y}$ on both sides of this equation,

$$
\begin{aligned}
& H_{x} H_{y 0}{ }^{C} D_{t}^{\alpha_{1}} u\left(x_{i}, y_{j}, t_{n}\right)+H_{x} H_{y 0}{ }^{C} D_{t}^{\alpha} u\left(x_{i}, y_{j}, t_{n}\right) \\
= & H_{x} H_{y}\left(u_{x x}\left(x_{i}, y_{j}, t_{n}\right)+u_{y y}\left(x_{i}, y_{j}, t_{n}\right)\right)+H_{x} H_{y} f\left(x_{i}, y_{j}, t_{n}\right) \mathrm{t}, \quad\left(x_{i}, y_{j}\right) \in \Omega_{h}, 1 \leq n \leq N .
\end{aligned}
$$

From Lemmas 2.1 and 2.2,

$$
\begin{array}{r}
H_{x} H_{y} D_{\tau}^{\alpha_{1}} U_{i j}^{n}+H_{x} H_{y} D_{\tau}^{\alpha} U_{i j}^{n}=H_{y} \delta_{x}^{2} U_{i j}^{n}+H_{x} \delta_{y}^{2} U_{i j}^{n}+H_{x} H_{y} f_{i j}^{n}+\bar{R}_{i j}^{n}, \\
\left(x_{i}, y_{j}\right) \in \Omega_{h}, 1 \leq n \leq N,
\end{array}
$$

where

$$
\begin{aligned}
\bar{R}_{i j}^{n}= & \frac{h_{1}^{4}}{360} \int_{0}^{1}\left[\frac{\partial^{6} u}{\partial x^{6}}\left(x_{i}-\lambda h_{1}, y_{j}, t_{n}\right)+\frac{\partial^{6} u}{\partial x^{6}}\left(x_{i}+\lambda h_{1}, y_{j}, t_{n}\right)\right] \zeta(\lambda) d \lambda \\
& +\frac{h_{2}^{4}}{360} \int_{0}^{1}\left[\frac{\partial^{6} u}{\partial y^{6}}\left(x_{i}, y_{j}-\lambda h_{2}, t_{n}\right)+\frac{\partial^{6} u}{\partial y^{6}}\left(x_{i}, y_{j}+\lambda h_{2}, t_{n}\right)\right] \zeta(\lambda) d \lambda \\
& +H_{x} H_{y} R^{\alpha_{1}}\left(u\left(x_{i}, t_{k}\right)\right)+H_{x} H_{y} R^{\alpha}\left(u\left(x_{i}, t_{k}\right)\right)
\end{aligned}
$$


Adding a small term $\frac{\mu_{1} \mu_{0} \tau}{\mu_{1}+\mu_{0}} \delta_{x}^{2} \delta_{y}^{2} \delta_{t} U_{i j}^{n-\frac{1}{2}}$ into Eq. (5.4), we then have

$$
\begin{aligned}
& H_{x} H_{y} D_{\tau}^{\alpha_{1}} U_{i j}^{n}+H_{x} H_{y} D_{\tau}^{\alpha} U_{i j}^{n}+\frac{\mu_{1} \mu_{0} \tau}{\mu_{1}+\mu_{0}} \delta_{x}^{2} \delta_{y}^{2} \delta_{t} U_{i j}^{n-\frac{1}{2}} \\
= & H_{y} \delta_{x}^{2} U_{i j}^{n}+H_{x} \delta_{y}^{2} U_{i j}^{n}+H_{x} H_{y} f_{i j}^{n}+R_{i j}^{n}, \quad\left(x_{i}, y_{j}\right) \in \Omega_{h}, 1 \leq n \leq N,
\end{aligned}
$$

where

$$
R_{i j}^{n}=\bar{R}_{i j}^{n}+\frac{\mu_{1} \mu_{0} \tau}{\mu_{1}+\mu_{0}} \delta_{x}^{2} \delta_{y}^{2} \delta_{t} U_{i j}^{n-\frac{1}{2}}
$$

so there exists a positive constant $c_{2}$ independent of $h_{1}, h_{2}$ and $\tau$ such that

$$
\left|R_{i j}^{n-\frac{1}{2}}\right| \leq c_{2}\left(\tau^{\min \{1+\alpha, 2-\alpha\}}+h_{1}^{4}+h_{2}^{4}\right), \quad\left(x_{i}, y_{j}\right) \in \Omega_{h}, 1 \leq n \leq N .
$$

In addition, on noting the initial and boundary value conditions (5.2)-(5.3) we find that

$$
\begin{aligned}
& U_{i j}^{0}=\varphi\left(x_{i}, y_{j}\right), \quad\left(x_{i}, y_{j}\right) \in \Omega_{h}, \\
& U_{i j}^{n}=\phi\left(x_{i}, y_{j}, t_{n}\right), \quad\left(x_{i}, y_{j}\right) \in \partial \Omega_{h}, \quad 1 \leq n \leq N .
\end{aligned}
$$

Omitting small terms $R_{i j}^{n}$ in Eq. (5.5), and replacing the function $U_{i j}^{n}$ with its numerical approximation $u_{i j}^{n}$ in Eqs. (5.5), (5.7) and (5.8), we obtain the following compact difference scheme (L1-CADI):

$$
\begin{aligned}
& H_{x} H_{y} D_{\tau}^{\alpha_{1}} u_{i j}^{n}+H_{x} H_{y} D_{\tau}^{\alpha} u_{i j}^{n}+\frac{\mu_{1} \mu_{0} \tau}{\mu_{1}+\mu_{0}} \delta_{x}^{2} \delta_{y}^{2} \delta_{t} u_{i j}^{n-\frac{1}{2}} \\
& \quad=H_{y} \delta_{x}^{2} u_{i j}^{n}+H_{x} \delta_{y}^{2} u_{i j}^{n}+H_{x} H_{y} f_{i j}^{n}, \quad\left(x_{i}, y_{j}\right) \in \Omega_{h}, \quad 1 \leq n \leq N, \\
& u_{i j}^{0}=\varphi\left(x_{i}, y_{j}\right), \quad\left(x_{i}, y_{j}\right) \in \Omega_{h}, \\
& u_{i j}^{n}=\phi\left(x_{i}, y_{j}, t_{n}\right), \quad\left(x_{i}, y_{j}\right) \in \partial \Omega_{h}, \quad 1 \leq n \leq N .
\end{aligned}
$$

To efficiently solve this formulation, the following techniques can be used. Introducing the intermediate variable $u_{i j}^{*}$, we obtain the D'Yakonov-type ADI scheme

$$
\left\{\begin{array}{c}
{\left[H_{x}-\frac{\mu_{1} \mu_{0}}{\mu_{1}+\mu_{0}} \delta_{x}^{2}\right] u_{i j}^{*}=\left(\frac{\mu_{1} \mu_{0}}{\mu_{1}+\mu_{0}}\right)^{2} \delta_{x}^{2} \delta_{y}^{2} u_{i j}^{n-1}+\frac{\mu_{0}}{\mu_{1}+\mu_{0}} \sum_{k=1}^{n-1}\left(a_{n-k-1}^{\alpha_{1}}-a_{n-k}^{\alpha_{1}}\right) H_{x} H_{y} u_{i j}^{k}} \\
\quad+\frac{\mu_{0} a_{n-1}^{\alpha_{1}}}{\mu_{1}+\mu_{0}} H_{x} H_{y} \psi_{i j}+\frac{\mu_{1}}{\mu_{1}+\mu_{0}} \sum_{k=1}^{n-1}\left(a_{n-k-1}^{\alpha}-a_{n-k}^{\alpha}\right) H_{x} H_{y} u_{i j}^{k} \\
\quad+\frac{\mu_{0} a_{n-1}^{\alpha}}{\mu_{1}+\mu_{0}} H_{x} H_{y} \psi_{i j}+\frac{\mu_{1} \mu_{0}}{\mu_{1}+\mu_{0}} H_{x} H_{y} f_{i j}^{n}, \\
{\left[H_{y}-\frac{\mu_{1} \mu_{0}}{\mu_{1}+\mu_{0}} \delta_{y}^{2}\right] u_{i j}^{n}=u_{i j}^{*} .}
\end{array}\right.
$$


We determine $\left\{u_{i j}^{n}\right\}$ by solving two sets of independent one-dimensional problems. Since the coefficient matrices are strictly diagonally dominant, at each time level one can run the $x$-sweep of the procedure to compute $\left\{u_{i j}^{*} \mid\left(x_{i}, y_{j}\right) \in \Omega_{h}\right\}$ with the known data $\left\{u_{i j}^{n-1} \mid\left(x_{i}, y_{j}\right)\right.$ $\left.\in \Omega_{h}\right\}$ using the fast tridiagonal (Thomas) algorithm - cf. Ref. [35] for example. Then once the solution $\left\{u_{i j}^{*} \mid\left(x_{i}, y_{j}\right) \in \Omega_{h}\right\}$ is available, the $y$-sweep can readily be performed to calculate the desired unique solution $\left\{u_{i j}^{n} \mid\left(x_{i}, y_{j}\right) \in \Omega_{h}\right\}$ to the compact ADI difference scheme (5.9)-(5.11).

\subsection{Stability and convergence of the L1-CADI scheme}

To analyse the stability and convergence of the L1-CADI scheme (5.9)-(5.11), we introduce some important lemmas.

Lemma 5.1. If $0<\gamma<1$, for any grid function $\left\{u_{i j}^{n} \mid\left(x_{i}, y_{j}\right) \in \bar{\Omega}_{h}, 0 \leq n \leq N\right\}$ defined on $\bar{\Omega}_{h} \times \Omega_{\tau}$ we have

$$
\begin{aligned}
-\left(H_{x} H_{y} D_{\tau}^{\gamma} u^{n}, \Delta_{h} u^{n}\right)= & \frac{1}{\mu}\left[\left\|\nabla_{h} u^{n}\right\|_{h^{*}}^{2}-\sum_{k=1}^{n-1}\left(a_{n-k-1}^{\gamma}-a_{n-k}^{\gamma}\right)\left\langle\nabla_{h} u^{k}, \nabla_{h} u^{n}\right\rangle_{h^{*}}\right. \\
& \left.-a_{k-1}^{\gamma}\left\langle\nabla_{h} u^{0}, \nabla_{h} u^{n}\right\rangle_{h^{*}}\right] .
\end{aligned}
$$

Proof. The result follows from the definition of $\|\cdot\|_{h^{*}}$ and the discrete Green formula.

Lemma 5.2. For any grid function $u \in V_{h}^{*}$,

$$
\left(\left(H_{y} \delta_{x}^{2}+H_{x} \delta_{y}^{2}\right) u, \Delta_{h} u\right) \geq \frac{2}{3}\left\|\Delta_{h} u\right\|^{2} .
$$

Proof. From the discrete Green formulation,

$$
\begin{aligned}
& \left(\left(H_{y} \delta_{x}^{2}+H_{x} \delta_{y}^{2}\right) u, \Delta_{h} u\right) \\
= & \left(\left(I+\frac{h_{2}^{2}}{12} \delta_{y}^{2}\right) \delta_{x}^{2} u+\left(I+\frac{h_{1}^{2}}{12} \delta_{x}^{2}\right) \delta_{y}^{2} u, \Delta_{h} u\right) \\
= & \left\|\Delta_{h} u\right\|^{2}-\frac{h_{1}^{2}}{12}\left(\left\|\delta_{x} \delta_{y}^{2} u\right\|^{2}+\left\|\delta_{x}^{2} \delta_{y} u\right\|^{2}\right)-\frac{h_{2}^{2}}{12}\left(\left\|\delta_{x} \delta_{y}^{2} u\right\|^{2}+\left\|\delta_{x}^{2} \delta_{y} u\right\|^{2}\right) \\
\geq & \left\|\Delta_{h} u\right\|^{2}-\frac{1}{3}\left(\left\|\delta_{y}^{2} u\right\|^{2}+\left\|\delta_{x} \delta_{y} u\right\|^{2}\right)-\frac{1}{3}\left(\left\|\delta_{x}^{2} u\right\|^{2}+\left\|\delta_{x} \delta_{y} u\right\|^{2}\right) \\
\geq & \frac{2}{3}\left\|\Delta_{h} u\right\|^{2},
\end{aligned}
$$

where we have used

$$
\begin{array}{rlrl}
\left\|\delta_{x} \delta_{y} u\right\| & \leq 2 h_{1}^{-1}\left\|\delta_{y} u\right\|, & & \left\|\delta_{x}^{2} u\right\| \leq 2 h_{1}^{-1}\left\|\delta_{x} u\right\|, \\
\left\|\delta_{x} \delta_{y} u\right\| \leq 2 h_{2}^{-1}\left\|\delta_{x} u\right\|, & \left\|\delta_{y}^{2} u\right\| \leq 2 h_{2}^{-1}\left\|\delta_{y} u\right\| .
\end{array}
$$


Lemma 5.3. For any grid function $u \in V_{h}^{*}$, we have

$$
\frac{1}{3}\left\|\nabla_{h} u\right\|^{2} \leq\left\|\nabla_{h} u\right\|_{h^{*}}^{2} \leq\left\|\nabla_{h} u\right\|^{2} .
$$

Proof. The result readily follows from the definition of $\|\cdot\|_{h^{*}}$ and inverse estimates.

We may now obtain an a priori estimate in the following Theorem.

Theorem 5.1. Let $\left\{v_{i j}^{n} \mid\left(x_{i}, y_{j}\right) \in \Omega_{h}, 0 \leq n \leq N\right\}$ be the solution of the difference system

$$
\begin{aligned}
& H_{x} H_{y} D_{\tau}^{\alpha_{1}} v_{i j}^{n}+H_{x} H_{y} D_{\tau}^{\alpha} v_{i j}^{n}+\frac{\mu_{1} \mu_{0} \tau}{\mu_{1}+\mu_{0}} \delta_{x}^{2} \delta_{y}^{2} \delta_{t} v_{i j}^{n-\frac{1}{2}} \\
& \quad=H_{y} \delta_{x}^{2} v_{i j}^{n}+H_{x} \delta_{y}^{2} v_{i j}^{n}+g_{i j}^{n}, \quad\left(x_{i}, y_{j}\right) \in \Omega_{h}, 1 \leq n \leq N, \\
& v_{i j}^{0}=v^{0}\left(x_{i}, y_{j}\right), \quad\left(x_{i}, y_{j}\right) \in \Omega_{h}, \\
& v_{i j}^{n}=0, \quad\left(x_{i}, y_{j}\right) \in \partial \Omega_{h}, \quad 1 \leq n \leq N,
\end{aligned}
$$

where $v^{0}\left(x_{i}, y_{j}\right) \equiv 0$ when $\left(x_{i}, y_{j}\right) \in \partial \Omega_{h}$. Then it follows that

$$
\begin{aligned}
\tau \sum_{k=1}^{n}\left\|\nabla_{h} v^{k}\right\|_{h^{*}}^{2} \leq & c_{\max }\left\{\frac{\mu_{1} \mu_{0} T}{\mu_{1}+\mu_{0}}\left(\left\|\delta_{x} \delta_{y}^{2} v^{0}\right\|^{2}+\left\|\delta_{x}^{2} \delta_{y} v^{0}\right\|^{2}\right)\right. \\
& \left.+\left(\frac{T^{1-\alpha_{1}}}{\Gamma\left(2-\alpha_{1}\right)}+\frac{T^{1-\alpha}}{\Gamma(2-\alpha)}\right)\left\|\nabla_{h} v^{0}\right\|_{h^{*}}^{2}+\frac{3}{4} \tau \sum_{k=1}^{n}\left\|g^{k}\right\|_{0}^{2}\right\}
\end{aligned}
$$

Proof. Taking the inner product of Eq. (5.12) with $-\Delta_{h} v^{n}$, we have

$$
\begin{aligned}
& -\left(H_{x} H_{y} D_{\tau}^{\alpha_{1}} v^{n}, \Delta_{h} v^{n}\right)-\left(H_{x} H_{y} D_{\tau}^{\alpha} v^{n}, \Delta_{h} v^{n}\right)-\frac{\mu_{1} \mu_{0} \tau}{\mu_{1}+\mu_{0}}\left(\delta_{x}^{2} \delta_{y}^{2} \delta_{t} v^{n-\frac{1}{2}}, \Delta_{h} v^{n}\right) \\
= & -\left(\left(H_{y} \delta_{x}^{2}+H_{x} \delta_{y}^{2}\right) v^{n}, \Delta_{h} v^{n}\right)-\left(g^{k}, \Delta_{h} v^{n}\right), \quad 1 \leq k \leq N .
\end{aligned}
$$

On using the discrete Green formula and Cauchy-Schwarz inequality, we rewrite the third term on the left-hand side of Eq. (5.15) as

$$
\begin{aligned}
& -\tau\left(\delta_{x}^{2} \delta_{y}^{2} \delta_{t} v^{n-\frac{1}{2}}, \Delta_{h} v^{n}\right) \\
= & -\left(\delta_{x}^{2} \delta_{y}^{2} v^{n}, \Delta_{h} v^{n}\right)+\left(\delta_{x}^{2} \delta_{y}^{2} v^{n-1}, \Delta_{h} v^{n}\right) \\
= & \left(\left\|\delta_{x}^{2} \delta_{y} v^{n}\right\|^{2}+\left\|\delta_{x} \delta_{y}^{2} v^{n}\right\|^{2}\right)-\left[\left(\delta_{x} \delta_{y}^{2} v^{n-1}, \delta_{x} \delta_{y}^{2} v^{n}\right)+\left(\delta_{x}^{2} \delta_{y} v^{n-1}, \delta_{x}^{2} \delta_{y} v^{n}\right)\right] \\
\geq & \left(\left\|\delta_{x}^{2} \delta_{y} v^{n}\right\|^{2}+\left\|\delta_{x} \delta_{y}^{2} v^{n}\right\|^{2}\right)-\frac{1}{2}\left[\left(\left\|\delta_{x} \delta_{y}^{2} v^{n-1}\right\|^{2}+\left\|\delta_{x} \delta_{y}^{2} v^{n}\right\|^{2}\right)\right. \\
& \left.+\left(\left\|\delta_{x}^{2} \delta_{y} v^{n-1}\right\|^{2}+\left\|\delta_{x}^{2} \delta_{y} v^{n}\right\|^{2}\right)\right] \\
\geq & \frac{1}{2}\left[\left(\left\|\delta_{x}^{2} \delta_{y} v^{n}\right\|^{2}+\left\|\delta_{x} \delta_{y}^{2} v^{n}\right\|^{2}\right)-\left(\left\|\delta_{x}^{2} \delta_{y} v^{n-1}\right\|^{2}+\left\|\delta_{x} \delta_{y}^{2} v^{n-1}\right\|^{2}\right)\right] .
\end{aligned}
$$


On substituting Eq. (5.16) into Eq. (5.15), and using Lemmas 5.1 and 5.2 together with the Cauchy-Schwarz inequality, we deduce that

$$
\begin{aligned}
& \left(\frac{1}{\mu_{1}}+\frac{1}{\mu_{0}}\right)\left\|\nabla_{h} v^{n}\right\|_{h^{*}}^{2}+\frac{2}{3}\left\|\Delta_{h} v^{n}\right\|^{2}+\frac{\mu_{1} \mu_{0}}{2\left(\mu_{1}+\mu_{0}\right)}\left[\left(\left\|\delta_{x}^{2} \delta_{y} v^{n}\right\|^{2}+\left\|\delta_{x} \delta_{y}^{2} v^{n}\right\|^{2}\right)\right. \\
& \left.-\left(\left\|\delta_{x}^{2} \delta_{y} v^{n-1}\right\|^{2}+\left\|\delta_{x} \delta_{y}^{2} v^{n-1}\right\|^{2}\right)\right] \\
\leq & \frac{1}{\mu_{1}} \sum_{k=1}^{n-1}\left(a_{n-k-1}^{\alpha_{1}}-a_{n-k}^{\alpha_{1}}\right)\left\langle\nabla_{h} v^{k}, \nabla_{h} v^{n}\right\rangle_{h^{*}}+\frac{a_{n-1}^{\alpha_{1}}}{\mu_{1}}\left\langle\nabla_{h} v^{0}, \nabla_{h} v^{n}\right\rangle_{h^{*}} \\
& +\frac{1}{\mu_{0}} \sum_{k=1}^{n-1}\left(a_{n-k-1}^{\alpha}-a_{n-k}^{\alpha}\right)\left\langle\nabla_{h} v^{k}, \nabla_{h} v^{n}\right\rangle_{h^{*}}+\frac{a_{n-1}^{\alpha}}{\mu_{0}}\left\langle\nabla_{h} v^{0}, \nabla_{h} v^{n}\right\rangle_{h^{*}}-\left(g^{n}, \nabla_{h} v^{n}\right) \\
\leq & \frac{1}{2 \mu_{1}} \sum_{k=1}^{n-1}\left(a_{n-k-1}^{\alpha_{1}}-a_{n-k}^{\alpha_{1}}\right)\left(\left\|\nabla_{h} v^{k}\right\|_{h^{*}}^{2}+\left\|\nabla_{h} v^{n}\right\|_{h^{*}}^{2}\right)+\frac{a_{k-1}^{\alpha_{1}}}{2 \mu_{1}}\left\|\nabla_{h} v^{0}\right\|_{h^{*}}^{2}+\frac{a_{k-1}^{\alpha_{1}}}{2 \mu_{1}}\left\|\nabla_{h} v^{n}\right\|_{h^{*}}^{2} \\
& +\frac{1}{2 \mu_{0}} \sum_{k=1}^{n-1}\left(a_{n-k-1}^{\alpha}-a_{n-k}^{\alpha}\right)\left(\left\|\nabla_{h} v^{k}\right\|_{h^{*}}^{2}+\left\|\nabla_{h} v^{n}\right\|_{h^{*}}^{2}\right)+\frac{a_{n-1}^{\alpha}}{2 \mu_{0}}\left\|\nabla_{h} v^{0}\right\|_{h^{*}}^{2}+\frac{a_{n-1}^{\alpha}}{2 \mu_{0}}\left\|\nabla_{h} v^{n}\right\|_{h^{*}}^{2} \\
& +\frac{3}{8}\left\|g^{n}\right\|^{2}+\frac{2}{3}\left\|\Delta_{h} v^{n}\right\|^{2},
\end{aligned}
$$

implying that

$$
\begin{aligned}
& \left(\frac{1}{\mu_{1}}+\frac{1}{\mu_{0}}\right)\left\|\nabla_{h} v^{n}\right\|_{h^{*}}^{2}+\frac{\mu_{1} \mu_{0}}{\mu_{1}+\mu_{0}}\left[\left(\left\|\delta_{x}^{2} \delta_{y} v^{n}\right\|^{2}+\left\|\delta_{x} \delta_{y}^{2} v^{n}\right\|^{2}\right)\right. \\
& \left.-\left(\left\|\delta_{x}^{2} \delta_{y} v^{n-1}\right\|^{2}+\left\|\delta_{x} \delta_{y}^{2} v^{n-1}\right\|^{2}\right)\right] \\
\leq & \sum_{k=1}^{n-1}\left[\frac{1}{\mu_{1}}\left(a_{n-k-1}^{\alpha_{1}}-a_{n-k}^{\alpha_{1}}\right)+\frac{1}{\mu_{0}}\left(a_{n-k-1}^{\alpha}-a_{n-k}^{\alpha}\right)\right]\left\|\nabla_{h} v^{k}\right\|_{h^{*}}^{2} \\
& +\left(\frac{a_{n-1}^{\alpha_{1}}}{\mu_{1}}+\frac{a_{n-1}^{\alpha}}{\mu_{0}}\right)\left\|\nabla_{h} v^{0}\right\|_{h^{*}}^{2}+\frac{3}{4}\left\|g^{n}\right\| .
\end{aligned}
$$

On denoting

$$
G^{n}=\sum_{k=1}^{n-1}\left(\frac{a_{n-k}^{\alpha_{1}}}{\mu_{1}}+\frac{a_{n-k}^{\alpha}}{\mu_{0}}\right)\left\|\nabla_{h} v^{k}\right\|_{h^{*}}^{2}+\frac{\mu_{1} \mu_{0}}{\mu_{1}+\mu_{0}}\left(\left\|\delta_{x}^{2} \delta_{y} v^{n}\right\|^{2}+\left\|\delta_{x} \delta_{y}^{2} v^{n}\right\|^{2}\right), \quad 1 \leq n \leq N
$$

and

$$
G^{0}=\frac{\mu_{1} \mu_{0}}{\mu_{1}+\mu_{0}}\left(\left\|\delta_{x}^{2} \delta_{y} v^{0}\right\|^{2}+\left\|\delta_{x} \delta_{y}^{2} v^{0}\right\|^{2}\right)
$$


Eq. (5.17) can be written as

$$
\begin{aligned}
G^{n} & \leq G^{n-1}+\left(\frac{a_{n-1}^{\alpha_{1}}}{\mu_{1}}+\frac{a_{n-1}^{\alpha}}{\mu_{0}}\right)\left\|\nabla_{h} v^{0}\right\|_{h^{*}}^{2}+\frac{3}{4}\left\|g^{n}\right\|^{2} \\
& \leq G^{0}+\sum_{k=1}^{n}\left(\frac{a_{k-1}^{\alpha_{1}}}{\mu_{1}}+\frac{a_{k-1}^{\alpha}}{\mu_{0}}\right)\left\|\nabla_{h} v^{0}\right\|_{h^{*}}^{2}+\frac{3}{4} \sum_{k=1}^{n}\left\|g^{k}\right\|^{2} .
\end{aligned}
$$

On the one hand, noting that $a_{n-k}^{\gamma} \geq(1-\gamma)(n-k+1)^{1-\gamma} \geq(1-\gamma) N^{-\gamma}$ where $\gamma=\alpha_{1}, \alpha$ we arrive at

$$
\begin{aligned}
& \frac{a_{n-k}^{\alpha_{1}}}{\mu_{1}}=\frac{a_{n-k}^{\alpha_{1}}}{\tau^{\alpha_{1}} \Gamma\left(2-\alpha_{1}\right)} \geq \frac{\left(1-\alpha_{1}\right) N^{-\alpha_{1}}}{\tau^{\alpha_{1}} \Gamma\left(2-\alpha_{1}\right)}=\frac{1}{T^{\alpha_{1}} \Gamma\left(1-\alpha_{1}\right)}, \\
& \frac{a_{n-k}^{\alpha}}{\mu_{0}}=\frac{a_{n-k}^{\alpha}}{\tau^{\alpha} \Gamma(2-\alpha)} \geq \frac{(1-\alpha) N^{-\alpha}}{\tau^{\alpha} \Gamma(2-\alpha)}=\frac{1}{T^{\alpha} \Gamma(1-\alpha)} .
\end{aligned}
$$

On the other hand, noting that $a_{k}^{\gamma}=(k+1)^{1-\gamma}-k^{1-\gamma}$ we have

$$
\begin{aligned}
& \tau \sum_{k=1}^{n} \frac{a_{k-1}^{\alpha_{1}}}{\mu_{1}} \leq \frac{\tau n^{1-\alpha_{1}}}{\tau^{\alpha_{1}} \Gamma\left(2-\alpha_{1}\right)} \leq \frac{t_{n}^{1-\alpha_{1}}}{\Gamma\left(2-\alpha_{1}\right)} \leq \frac{T^{1-\alpha_{1}}}{\Gamma\left(2-\alpha_{1}\right)}, \\
& \tau \sum_{k=1}^{n} \frac{a_{k-1}^{\alpha}}{\mu_{0}} \leq \frac{\tau n^{1-\alpha}}{\tau^{\alpha} \Gamma(2-\alpha)} \leq \frac{t_{n}^{1-\alpha}}{\Gamma(2-\alpha)} \leq \frac{T^{1-\alpha}}{\Gamma(2-\alpha)} .
\end{aligned}
$$

Multiplying Eq. (5.18) by $\tau$ and invoking the above inequality yields the desired result.

From Theorem 5.1, we obtain the following stability statement.

Theorem 5.2. The L1-CADI scheme (5.9)-(5.11) is unconditionally stable to the initial value $\varphi$ and the inhomogeneous term $f$.

Let us now consider the convergence of the L1-CADI scheme (5.9)-(5.11).

Theorem 5.3. Assume that $u(x, y, t) \in C_{x, y, t}^{6,6,2}(\Omega \times[0, T])$ is the solution of Eqs. (5.1)-(5.3), and $\left\{u_{i j}^{n} \mid\left(x_{i}, y_{j}\right) \in \Omega_{h}, 0 \leq n \leq N\right\}$ is the solution of the L1-CADI scheme (5.9)-(5.11). Then

$$
\sqrt{\tau \sum_{k=1}^{n}\left\|\nabla_{h} e^{k}\right\|^{2}} \leq \frac{3 c_{2}}{2} \sqrt{T L_{1} L_{2}}\left(\tau^{\min \{1+\alpha, 2-\alpha\}}+h_{1}^{4}+h_{2}^{4}\right), \quad 1 \leq n \leq N .
$$

Proof. Let

$$
e_{i j}^{n}=U_{i j}^{n}-u_{i j}^{n}, \quad\left(x_{i}, y_{j}\right) \in \bar{\Omega}_{h}, \quad 0 \leq n \leq N .
$$

Subtracting Eqs. (5.9)-(5.11) from Eqs. (5.5), (5.7) and (5.8) respectively, we obtain the error equations

$$
\begin{aligned}
& H_{x} H_{y} D_{\tau}^{\alpha_{1}} e_{i j}^{n}+H_{x} H_{y} D_{\tau}^{\alpha} e_{i j}^{n}+\frac{\mu_{1} \mu \tau}{\mu_{1}+\mu} \delta_{x}^{2} \delta_{y}^{2} \delta_{t} e_{i j}^{n-\frac{1}{2}} \\
& \quad=H_{y} \delta_{x}^{2} e_{i j}^{n}+H_{x} \delta_{y}^{2} e_{i j}^{n}+R_{i j}^{n}, \quad\left(x_{i}, y_{j}\right) \in \Omega_{h}, 1 \leq n \leq N, \\
& e_{i j}^{0}=0, \quad\left(x_{i}, y_{j}\right) \in \Omega_{h}, \\
& e_{i j}^{n}=0, \quad\left(x_{i}, y_{j}\right) \in \partial \Omega_{h}, \quad 0 \leq n \leq N,
\end{aligned}
$$


where $D_{\tau}^{\gamma} e_{i j}^{n}=D_{\tau}^{\gamma}\left(U_{i j}^{n}-u_{i j}^{n}\right)$ and $\gamma=\alpha, \alpha_{1}$. Using inequality (5.6), it follows from Theorem 5.1 and Lemma 5.3 that

$$
\begin{aligned}
\tau \sum_{k=1}^{n}\left\|\nabla_{h} e^{k}\right\|^{2} & \leq \frac{9 \tau}{4} \sum_{k=1}^{n}\left\|R^{k}\right\|^{2} \leq \frac{9 \tau}{4} \sum_{k=1}^{n} L_{1} L_{2} c_{2}^{2}\left(\tau^{\min \{1+\alpha, 2-\alpha\}}+h_{1}^{4}+h_{2}^{4}\right)^{2} \\
& \leq \frac{9 T}{4} c_{2}^{2} L_{1} L_{2}\left(\tau^{\min \{1+\alpha, 2-\alpha\}}+h_{1}^{4}+h_{2}^{4}\right)^{2}, \quad 1 \leq n \leq N .
\end{aligned}
$$

\section{A Second-Order ADI Finite Difference Scheme for the 2D Problem}

We consider a difference scheme L1-ADI for the problem defined by Eqs. (5.1)-(5.3) as follows:

$$
\begin{aligned}
& D_{\tau}^{\alpha_{1}} u_{i j}^{n}+D_{\tau}^{\alpha} u_{i j}^{n}+\frac{\mu_{1} \mu_{0} \tau}{\mu_{1}+\mu_{0}} \delta_{x}^{2} \delta_{y}^{2} \delta_{t} u_{i j}^{n-\frac{1}{2}} \\
& \quad=\Delta_{h} u_{i j}^{n}+f_{i j}^{n}, \quad\left(x_{i}, y_{j}\right) \in \bar{\Omega}_{h}, \quad 1 \leq n \leq N, \\
& u_{i j}^{0}=\varphi\left(x_{i}, y_{j}\right), \quad\left(x_{i}, y_{j}\right) \in \bar{\Omega}_{h}, \\
& u_{i j}^{n}=\phi\left(x_{i}, y_{j}, t_{n}\right), \quad\left(x_{i}, y_{j}\right) \in \partial \Omega_{h}, \quad 1 \leq n \leq N .
\end{aligned}
$$

The difference scheme for Eq. (6.1) can be written in the ADI form

$$
\left\{\begin{array}{l}
{\left[I-\frac{\mu_{1} \mu_{0}}{\mu_{1}+\mu_{0}} \delta_{x}^{2}\right] u_{i j}^{*}=\left(\frac{\mu_{1} \mu_{0}}{\mu_{1}+\mu_{0}}\right)^{2} \delta_{x}^{2} \delta_{y}^{2} u_{i j}^{n-1}+\frac{\mu_{0}}{\mu_{1}+\mu_{0}} \sum_{k=1}^{n-1}\left(a_{n-k-1}^{\alpha_{1}}-a_{n-k}^{\alpha_{1}}\right) u_{i j}^{k}} \\
\quad+\frac{\mu_{0} a_{n-1}^{\alpha_{1}}}{\mu_{1}+\mu_{0}} \psi_{i j}+\frac{\mu_{1}}{\mu_{1}+\mu_{0}} \sum_{k=1}^{n-1}\left(a_{n-k-1}^{\alpha}-a_{n-k}^{\alpha}\right) u_{i j}^{k}+\frac{\mu_{0} a_{n-1}^{\alpha}}{\mu_{1}+\mu_{0}} \psi_{i j}+\frac{\mu_{1} \mu_{0}}{\mu_{1}+\mu_{0}} f_{i j}^{n} \\
{\left[I-\frac{\mu_{1} \mu_{0}}{\mu_{1}+\mu_{0}} \delta_{y}^{2}\right] u_{i j}^{n}=u_{i j}^{*} .}
\end{array}\right.
$$

Lemma 6.1. Suppose that $u(x, y, t) \in C_{x, y, t}^{4,4,2}(\Omega \times[0, T])$ is the solution of the problem (5.1)(5.3). Then the truncation error of the L1-ADI scheme (6.1)-(6.3) satisfies

$$
\left|\tilde{R}_{i j}^{k}\right| \leq \tilde{c}_{R}\left(\tau^{\min \{1+\alpha, 2-\alpha\}}+h_{1}^{2}+h_{2}^{2}\right), \quad\left(x_{i}, y_{j}\right) \in \bar{\Omega}_{h}, 1 \leq n \leq N,
$$

where $\tilde{c}_{R}$ is a positive constant independent of $\tau$ and $h_{1}, h_{2}$.

The proofs for the stability and convergence for the L1-ADI scheme (6.1)-(6.3) are essentially the same as for Theorems 5.1 and 5.3, so we omit the details and simply state the relevant results. 
Theorem 6.1. The L1-ADI scheme (6.1)-(6.3) is unconditionally stable to the initial value $\varphi$ and the inhomogeneous term $f-$ i.e.

$$
\begin{aligned}
\tau \sum_{k=1}^{n}\left\|\nabla_{h} u^{k}\right\|^{2} \leq & c_{\max }\left\{\frac{\mu_{1} \mu_{0} T}{\mu_{1}+\mu_{0}}\left(\left\|\delta_{x} \delta_{y}^{2} u^{0}\right\|^{2}+\left\|\delta_{x}^{2} \delta_{y} u^{0}\right\|^{2}\right)\right. \\
& \left.+\left(\frac{T^{1-\alpha_{1}}}{\Gamma\left(2-\alpha_{1}\right)}+\frac{T^{1-\alpha}}{\Gamma(2-\alpha)}\right)\left\|\nabla_{h} u^{0}\right\|^{2}+\frac{3}{4} \tau \sum_{k=1}^{n}\left\|f^{k}\right\|^{2}\right\} .
\end{aligned}
$$

Theorem 6.2. Suppose $u(x, y, t)$ is the smooth solution of (5.1)-(5.3) and $\left\{u_{i j}^{n} \mid\left(x_{i}, y_{j}\right) \in\right.$ $\left.\bar{\Omega}_{h}, 0 \leq n \leq N\right\}$ is the solution of the L1-ADI scheme (6.1)-(6.3), respectively. Then

$$
\sqrt{\tau \sum_{k=1}^{n}\left\|\nabla_{h} e^{k}\right\|^{2}} \leq \frac{\tilde{c}_{R}}{2} \sqrt{3 T L_{1} L_{2}}\left(\tau^{\min \{1+\alpha, 2-\alpha\}}+h_{1}^{2}+h_{2}^{2}\right), \quad 1 \leq n \leq N .
$$

\section{Numerical Experiments}

We now present numerical solutions of the problem using the numerical methods previously discussed, where the errors involved are measured by comparing the numerical solutions with exact solutions.

Example 7.1. Let $L=\pi, T=1$. In order to test the convergence rate of the proposed methods, we refer to the exact solution of the problem (2.1)-(2.3) - viz.

$$
u(x, t)=t^{1+\alpha_{1}+\alpha} \sin x .
$$

It can readily be checked that the corresponding source term $f(x, t)$ and the initial and boundary conditions are respectively

$$
f(x, t)=\left(\frac{\Gamma\left(2+\alpha_{1}+\alpha\right)}{\Gamma(2+\alpha)} t^{2+\alpha}+\frac{\Gamma\left(2+\alpha_{1}+\alpha\right)}{\Gamma\left(2+\alpha_{1}\right)} t^{2+\alpha_{1}}+t^{1+\alpha_{1}+\alpha}\right) \sin x
$$

and

$$
\varphi(x)=0, \quad \psi_{1}(t)=0, \quad \psi_{2}(t)=0 .
$$

We compute the maximum norm errors of the numerical solution

$$
e_{\infty}(h, \tau)=\max _{0 \leq k \leq N}\left\|U^{k}-u^{k}\right\|_{\infty},
$$

and respectively characterise the temporal convergence order and the spatial convergence order as

$$
\operatorname{Order} 1=\log _{2}\left(\frac{e_{\infty}(h, 2 \tau)}{e_{\infty}(h, \tau)}\right), \quad \text { Order2 }=\log _{2}\left(\frac{e_{\infty}(2 h, \tau)}{e_{\infty}(h, \tau)}\right) .
$$

The first computational investigation concerns the temporal errors and convergence orders. In order to find the temporal convergence order, the space step $h$ should be chosen 
Table 1: Numerical convergence of the L1-CD and L1-SOD schemes in the temporal direction.

\begin{tabular}{|c|c|cc|cc|}
\hline & & \multicolumn{2}{|c|}{ the L1-CD scheme } & \multicolumn{2}{c|}{ the L1-SOD scheme } \\
$\alpha_{1}, \alpha$ & $\tau$ & $e_{\infty}(h, \tau)$ & Order1 & $e_{\infty}(h, \tau)$ & Order1 \\
\hline \multirow{3}{*}{$\alpha_{1}=0.15, \alpha=0.95$} & $1 / 10$ & $3.776 \mathrm{e}-2$ & $*$ & $3.776 \mathrm{e}-2$ & $*$ \\
& $1 / 20$ & $1.836 \mathrm{e}-2$ & 1.040 & $1.836 \mathrm{e}-2$ & 1.040 \\
& $1 / 40$ & $8.890 \mathrm{e}-3$ & 1.047 & $8.890 \mathrm{e}-3$ & 1.047 \\
& $1 / 80$ & $4.296 \mathrm{e}-3$ & 1.049 & $4.296 \mathrm{e}-3$ & 1.049 \\
& $1 / 160$ & $2.075 \mathrm{e}-3$ & 1.050 & $2.075 \mathrm{e}-3$ & 1.050 \\
\hline \multirow{3}{*}{$\alpha_{1}=0.35, \alpha=0.65$} & $1 / 10$ & $1.242 \mathrm{e}-2$ & $*$ & $1.242 \mathrm{e}-2$ & $*$ \\
& $1 / 20$ & $4.817 \mathrm{e}-3$ & 1.366 & $4.817 \mathrm{e}-3$ & 1.366 \\
& $1 / 40$ & $1.858 \mathrm{e}-3$ & 1.374 & $1.858 \mathrm{e}-3$ & 1.374 \\
& $1 / 80$ & $7.159 \mathrm{e}-4$ & 1.376 & $7.160 \mathrm{e}-4$ & 1.376 \\
& $1 / 160$ & $2.759 \mathrm{e}-4$ & 1.376 & $2.761 \mathrm{e}-4$ & 1.375 \\
\hline
\end{tabular}

Table 2: Numerical convergence of the L1-CD scheme in the spatial direction with $\tau=1 / 200000$.

\begin{tabular}{|c|ccc|}
\hline$\alpha_{1}, \alpha$ & $h$ & $e_{\infty}(h, \tau)$ & Order2 \\
\hline \multirow{3}{*}{$\alpha_{1}=0.35, \alpha=0.65$} & $\pi / 2$ & $6.413 \mathrm{e}-3$ & $*$ \\
& $\pi / 4$ & $3.788 \mathrm{e}-4$ & 4.082 \\
& $\pi / 8$ & $2.328 \mathrm{e}-5$ & 4.024 \\
& $\pi / 16$ & $1.464 \mathrm{e}-6$ & 3.991 \\
\hline \multirow{3}{*}{$\alpha_{1}=0.45, \alpha=0.55$} & $\pi / 2$ & $6.450 \mathrm{e}-3$ & $*$ \\
& $\pi / 4$ & $3.809 \mathrm{e}-4$ & 4.082 \\
& $\pi / 8$ & $2.340 \mathrm{e}-5$ & 4.025 \\
& $\pi / 16$ & $1.461 \mathrm{e}-6$ & 4.002 \\
\hline
\end{tabular}

sufficiently small to prevent the effect of the spatial discretisation error entering into the calculation. The computational results of the L1-CD scheme (2.8)-(2.10) and the L1-SOD scheme (4.1)-(4.3) with $h=\pi / 100$ and $h=\pi / 1000$ are presented in Table 1, respectively. It is observed that both schemes generate $2-\alpha$ temporal convergence order, consistent with our theoretical analysis.

Secondly, we test the spatial errors and convergence orders of the two schemes by letting $h$ vary, but fixing the time step $\tau$ sufficiently small to avoid contamination of the spatial error. Tables 2 and 3 show the maximum norm errors and spatial convergence orders of the L1-CD scheme and the L1-SOD scheme with different $\alpha_{1}, \alpha$. As predicted by our theoretical estimates, the L1-CD scheme attains fourth-order spatial accuracy while the L1-SOD scheme has second-order spatial accuracy.

Next, in order to quantify some features of the computational efficiencies of the L1-CD scheme more precisely, we investigate the CPU time of both schemes. As mentioned before, since fractional derivatives are non-local operators they require a large memory storage capacity if low-order finite difference methods are employed for the spatial approximation. For the L1-SOD scheme, the optimal step sizes satisfy $\tau^{2-\alpha} \approx h^{2}$, or $N \approx\left[M^{\frac{2}{2-\alpha}}\right]$. For the L1-CD scheme, the optimal step sizes satisfy $\tau^{2-\alpha} \approx h^{4}$, or $N \approx\left[M^{\frac{4}{2-\alpha}}\right]$.

From Table 4, it is clear that the two schemes provide almost the same accuracy for 
Table 3: Numerical convergence of the L1-SOD scheme in the spatial direction with $\tau=1 / 20000$.

\begin{tabular}{|c|ccc|}
\hline$\alpha_{1}, \alpha$ & $h$ & $e_{\infty}(h, \tau)$ & Order2 \\
\hline \multirow{3}{*}{$\alpha_{1}=0.35, \alpha=0.65$} & $\pi / 2$ & $4.612 \mathrm{e}-2$ & $*$ \\
& $\pi / 4$ & $1.188 \mathrm{e}-2$ & 1.956 \\
& $\pi / 8$ & $2.993 \mathrm{e}-3$ & 1.990 \\
& $\pi / 16$ & $7.497 \mathrm{e}-4$ & 1.997 \\
\hline \multirow{3}{*}{$\alpha_{1}=0.45, \alpha=0.55$} & $\pi / 2$ & $4.640 \mathrm{e}-2$ & $*$ \\
& $\pi / 4$ & $1.195 \mathrm{e}-2$ & 1.957 \\
& $\pi / 8$ & $3.009 \mathrm{e}-3$ & 1.990 \\
& $\pi / 16$ & $7.537 \mathrm{e}-4$ & 1.997 \\
\hline
\end{tabular}

Table 4: The maximum norm error and CPU time of the L1-CD and L1-SOD schemes.

\begin{tabular}{|c|c|ccc|ccc|}
\hline \multirow{2}{*}{$\alpha_{1}, \alpha$} & & \multicolumn{3}{|c|}{ the L1-CD scheme } & \multicolumn{3}{c|}{ the L1-SOD scheme } \\
$\alpha_{1}=0.1, \alpha=0.3$ & $N$ & $M$ & $e_{\infty}(h, \tau)$ & CPU time(s) & $M$ & $e_{\infty}(h, \tau)$ & CPU time(s) \\
& 225 & 10 & $1.678 \mathrm{e}-5$ & 0.1669 & 100 & $2.940 \mathrm{e}-5$ & 0.7005 \\
& 585 & 15 & $3.286 \mathrm{e}-6$ & 0.7557 & 225 & $5.802 \mathrm{e}-6$ & 4.8096 \\
& 1151 & 20 & $1.044 \mathrm{e}-6$ & 2.3417 & 400 & $1.835 \mathrm{e}-6$ & 20.8146 \\
& 1947 & 25 & $4.262 \mathrm{e}-7$ & 5.9645 & 625 & $7.512 \mathrm{e}-7$ & 70.2803 \\
\hline \multirow{3}{*}{$\alpha_{1}=0.1, \alpha=0.5$} & 464 & 10 & $2.159 \mathrm{e}-5$ & 0.3780 & 100 & $3.331 \mathrm{e}-5$ & 1.5963 \\
& 1368 & 15 & $4.229 \mathrm{e}-6$ & 2.1757 & 225 & $6.572 \mathrm{e}-6$ & 14.9198 \\
& 2947 & 20 & $1.343 \mathrm{e}-6$ & 8.7419 & 400 & $2.078 \mathrm{e}-6$ & 86.3564 \\
& 5344 & 25 & $5.485 \mathrm{e}-7$ & 28.4608 & 625 & $8.505 \mathrm{e}-7$ & 369.1998 \\
\hline \multirow{3}{*}{$=0.1, \alpha=0.7$} & 1194 & 10 & $3.070 \mathrm{e}-5$ & 1.1944 & 100 & $4.131 \mathrm{e}-5$ & 5.4915 \\
& 4157 & 15 & $6.024 \mathrm{e}-6$ & 11.1884 & 225 & $8.156 \mathrm{e}-6$ & 89.9025 \\
& 10073 & 20 & $1.916 \mathrm{e}-6$ & 65.8156 & 400 & $2.580 \mathrm{e}-6$ & 727.2762 \\
& 20015 & 25 & $7.829 \mathrm{e}-7$ & 292.5059 & 625 & $1.057 \mathrm{e}-6$ & 4146.7922 \\
\hline
\end{tabular}

the same temporal grid size, but the L1-CD scheme needs fewer spatial grid points and less $\mathrm{CPU}$ time. Thus the L1-CD scheme reduces both the storage requirement and the necessary CPU time successfully.

Finally, we compute the long time behaviour of the L1-CD and the L1-SOD schemes. The optimal step sizes again satisfy $\tau^{2-\alpha} \approx h^{2}$ for the L1-SOD scheme and $\tau^{2-\alpha} \approx h^{4}$ for the L1-CD scheme, on fixing $T=10$ and $M=4,6,8, \cdots, 24$. Fig. 1 shows the maximum error and CPU time of the L1-CD scheme and the L1-SOD scheme for $t=0,1,2, \cdots, 10$ when $\alpha_{1}=0.1, \alpha=0.2$, and the compact difference scheme efficiency.

Example 7.2. Let $T=1, \Omega=(0, \pi) \times(0, \pi)$. As before, we refer to the exact solution of the problem (5.1)-(5.3) - i.e. in this case

$$
u(x, y, t)=t^{3+\alpha_{1}+\alpha} \sin x \sin y .
$$

It is again not difficult to obtain the corresponding forcing term $f(x, y, t)$, and the initial and boundary conditions $\varphi(x, y)$ and $\psi(x, y, t)$.

In order to test the convergence rate of the proposed methods, we use the same spacing $h$ in each direction $\left(h=h_{1}=h_{2}\right.$ ), and compute the maximum norm errors of the numerical 

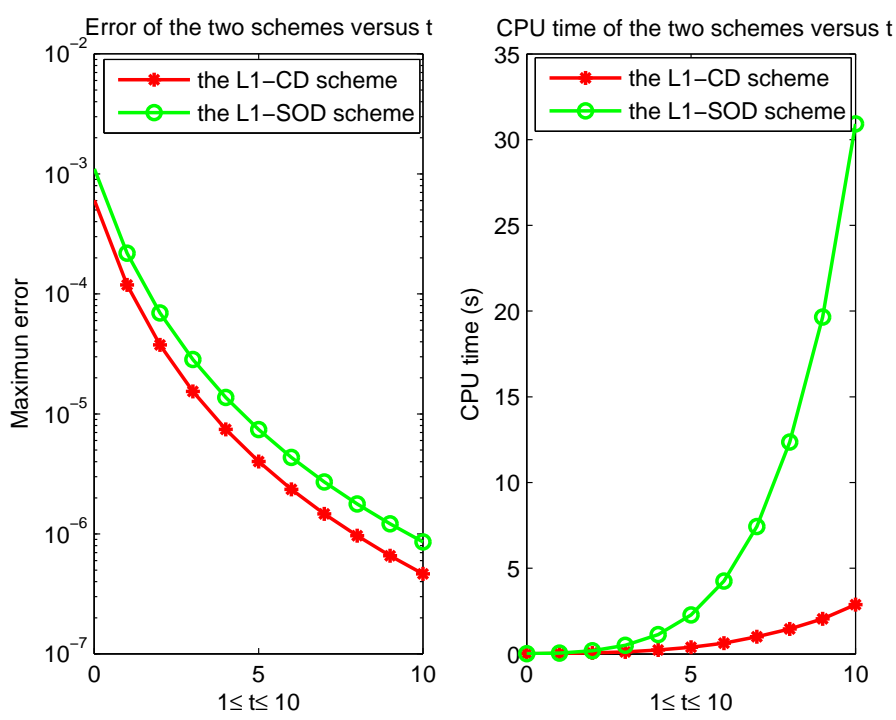

Figure 1: Error and CPU time of the L1-CD and L1-SOD schemes.

Table 5: Numerical convergence of the L1-CADI and L1-ADI schemes in the temporal direction.

\begin{tabular}{|c|c|cc|cc|}
\hline & & \multicolumn{2}{|c|}{ the L1-CADI scheme } & \multicolumn{2}{c|}{ the L1-ADI scheme } \\
$\alpha_{1}, \alpha$ & $\tau$ & $E_{\infty}(h, \tau)$ & Order1 & $E_{\infty}(h, \tau)$ & Order3 \\
\hline \multirow{3}{*}{$\alpha_{1}=0.2, \alpha=0.9$} & $1 / 10$ & $7.038 \mathrm{e}-2$ & $*$ & $7.038 \mathrm{e}-2$ & $*$ \\
& $1 / 20$ & $3.452 \mathrm{e}-2$ & 1.028 & $3.453 \mathrm{e}-2$ & 1.027 \\
& $1 / 40$ & $1.657 \mathrm{e}-2$ & 1.059 & $1.658 \mathrm{e}-2$ & 1.058 \\
& $1 / 80$ & $7.857 \mathrm{e}-3$ & 1.077 & $7.862 \mathrm{e}-3$ & 1.076 \\
& $1 / 160$ & $3.698 \mathrm{e}-3$ & 1.087 & $3.703 \mathrm{e}-3$ & 1.086 \\
\hline \multirow{3}{*}{$\alpha_{1}=0.5, \alpha=0.7$} & $1 / 10$ & $4.140 \mathrm{e}-2$ & $*$ & $4.141 \mathrm{e}-2$ & $*$ \\
& $1 / 20$ & $1.757 \mathrm{e}-2$ & 1.236 & $1.758 \mathrm{e}-2$ & 1.236 \\
& $1 / 40$ & $7.294 \mathrm{e}-3$ & 1.269 & $7.299 \mathrm{e}-3$ & 1.268 \\
& $1 / 80$ & $2.985 \mathrm{e}-3$ & 1.289 & $2.990 \mathrm{e}-3$ & 1.287 \\
& $1 / 160$ & $1.212 \mathrm{e}-3$ & 1.301 & $1.217 \mathrm{e}-3$ & 1.297 \\
\hline
\end{tabular}

solution

$$
E_{\infty}(h, \tau)=\max _{\substack{\left(x_{i}, y_{j}\right) \in \tilde{\Omega}_{h} \\ 0 \leq n \leq N}}\left|u\left(x_{i}, y_{j}, t_{n}\right)-u_{i j}^{n}\right|
$$

via

$$
\text { Order3 }=\log _{2}\left(\frac{E_{\infty}(h, 2 \tau)}{E_{\infty}(h, \tau)}\right), \text { Order } 4=\log _{2}\left(\frac{E_{\infty}(2 h, \tau)}{E_{\infty}(h, \tau)}\right)
$$

Firstly, the numerical accuracy in time is verified. For fixed space step sizes $h=\pi / 20$ and $h=\pi / 200$ respectively, and varying the temporal step size $\tau$, the computational results we obtain are displayed in Table 5. From this data, we conclude that there is $\min \{1+\alpha, 2-\alpha\}$-order convergence in time. 
Table 6: Numerical convergence of the L1-CADI scheme in the spatial direction with $\tau=1 / 200000$.

\begin{tabular}{|c|ccc|}
\hline$\alpha_{1}, \alpha$ & $h$ & $E_{\infty}(h, \tau)$ & Order4 \\
\hline \multirow{3}{*}{$\alpha_{1}=0.1, \alpha=0.2$} & $\pi / 2$ & $4.074 \mathrm{e}-3$ & $*$ \\
& $\pi / 4$ & $2.413 \mathrm{e}-4$ & 4.078 \\
& $\pi / 8$ & $1.482 \mathrm{e}-5$ & 4.025 \\
& $\pi / 16$ & $9.222 \mathrm{e}-7$ & 4.006 \\
\hline \multirow{3}{*}{$\alpha_{1}=0.2, \alpha=0.3$} & $\pi / 2$ & $2.768 \mathrm{e}-3$ & $*$ \\
& $\pi / 4$ & $1.641 \mathrm{e}-4$ & 4.076 \\
& $\pi / 8$ & $1.009 \mathrm{e}-5$ & 4.024 \\
& $\pi / 16$ & $6.334 \mathrm{e}-7$ & 3.993 \\
\hline
\end{tabular}

Table 7: Numerical convergence of the L1-ADI scheme in the spatial direction with $\tau=1 / 10000$.

\begin{tabular}{|c|ccc|}
\hline$\alpha_{1}, \alpha$ & $h$ & $E_{\infty}(h, \tau)$ & Order4 \\
\hline \multirow{3}{*}{$\alpha_{1}=0.1, \alpha=0.2$} & $\pi / 4$ & $2.314 \mathrm{e}-2$ & $*$ \\
& $\pi / 8$ & $5.769 \mathrm{e}-3$ & 2.004 \\
& $\pi / 16$ & $1.436 \mathrm{e}-3$ & 2.007 \\
& $\pi / 32$ & $3.528 \mathrm{e}-4$ & 2.025 \\
\hline \multirow{3}{*}{$\alpha_{1}=0.2, \alpha=0.3$} & $\pi / 4$ & $2.124 \mathrm{e}-2$ & $*$ \\
& $\pi / 8$ & $5.306 \mathrm{e}-3$ & 2.001 \\
& $\pi / 16$ & $1.324 \mathrm{e}-3$ & 2.003 \\
& $\pi / 32$ & $3.287 \mathrm{e}-4$ & 2.010 \\
\hline
\end{tabular}

Secondly, we test the spatial errors and convergence orders of the two schemes, by letting $h$ vary and fixing the time step $\tau$ sufficiently small in order to avoid significant contamination from the spatial error. Tables 6 and 7 give the maximum norm errors and spatial convergence orders for the two schemes. As predicted by the theoretical estimates, the L1-CADI scheme attains fourth-order spatial accuracy whereas the L1-ADI scheme has second-order spatial accuracy. In Table 8, we display some CPU time results for the L1CADI and L1-ADI schemes. It is clear that the two schemes generate almost the same accuracy for the same temporal grid size, while the L1-CADI scheme needs fewer spatial grid points and less CPU time, so it requites less storage and CPU time.

Similar to Example 7.1, we compute the problem for a longer time by fixing $T=10$ and $M=4,5,6, \cdots, 14$, and still choosing the optimal step size $\tau^{\min \{1+\alpha, 2-\alpha\}} \approx h^{2}$ for the L1-ADI scheme and $\tau^{\min \{1+\alpha, 2-\alpha\}} \approx h^{4}$ for the L1-CADI scheme, respectively. Fig. 2 shows the maximum error and CPU time of both schemes for $t=0,1,2, \cdots, 10$ when $\alpha_{1}=0.1$, $\alpha=0.2$, and also the efficiency of the L1-CADI scheme.

\section{Conclusions}

In this article, we discuss some computationally effective numerical methods for solving $1 \mathrm{D}$ and 2D multi-term time fractional sub-diffusion equations. Based on the $L 1$ approximation for the multi-term time Caputo fractional derivative in the temporal direction, and in order to reduce the storage requirement, a compact difference method for spatial 
Table 8: The maximum norm error and CPU time of the L1-CADI and L1-ADI schemes.

\begin{tabular}{|c|c|ccc|ccc|}
\hline & & \multicolumn{3}{|c|}{ the L1-CADI scheme } & \multicolumn{3}{c|}{ the L1-ADI scheme } \\
$\alpha_{1}, \alpha$ & $N$ & $M$ & $E_{\infty}(h, \tau)$ & CPU time(s) & $M$ & $E_{\infty}(h, \tau)$ & CPU time(s) \\
\hline \multirow{3}{*}{$\alpha_{1}=0.1, \alpha=0.3$} & 26 & 4 & $8.013 \mathrm{e}-6$ & 0.0952 & 16 & $8.406 \mathrm{e}-6$ & 0.1933 \\
& 68 & 6 & $6.330 \mathrm{e}-7$ & 0.4022 & 36 & $6.355 \mathrm{e}-7$ & 2.3234 \\
& 133 & 8 & $8.503 \mathrm{e}-8$ & 1.7451 & 64 & $8.510 \mathrm{e}-8$ & 15.2556 \\
& 225 & 10 & $1.659 \mathrm{e}-8$ & 5.9884 & 100 & $1.660 \mathrm{e}-8$ & 68.3041 \\
\hline \multirow{3}{*}{$\alpha_{1}=0.1, \alpha=0.5$} & 40 & 4 & $9.825 \mathrm{e}-6$ & 0.0979 & 16 & $9.861 \mathrm{e}-6$ & 0.2762 \\
& 119 & 6 & $2.380 \mathrm{e}-7$ & 0.7966 & 36 & $2.381 \mathrm{e}-7$ & 4.3325 \\
& 256 & 8 & $1.658 \mathrm{e}-8$ & 4.4757 & 64 & $1.658 \mathrm{e}-8$ & 33.2143 \\
& 464 & 10 & $2.056 \mathrm{e}-9$ & 18.5542 & 100 & $2.057 \mathrm{e}-9$ & 179.8494 \\
\hline \multirow{3}{*}{$\alpha_{1}=0.1, \alpha=0.7$} & 71 & 4 & $1.743 \mathrm{e}-6$ & 0.1580 & 16 & $1.744 \mathrm{e}-6$ & 0.4916 \\
& 248 & 6 & $1.668 \mathrm{e}-8$ & 2.1985 & 36 & $1.669 \mathrm{e}-8$ & 10.0185 \\
& 601 & 8 & $5.994 \mathrm{e}-10$ & 17.3999 & 64 & $5.994 \mathrm{e}-10$ & 100.2059 \\
& 1194 & 10 & $4.492 \mathrm{e}-11$ & 95.8941 & 100 & $4.492 \mathrm{e}-11$ & 917.2806 \\
\hline
\end{tabular}
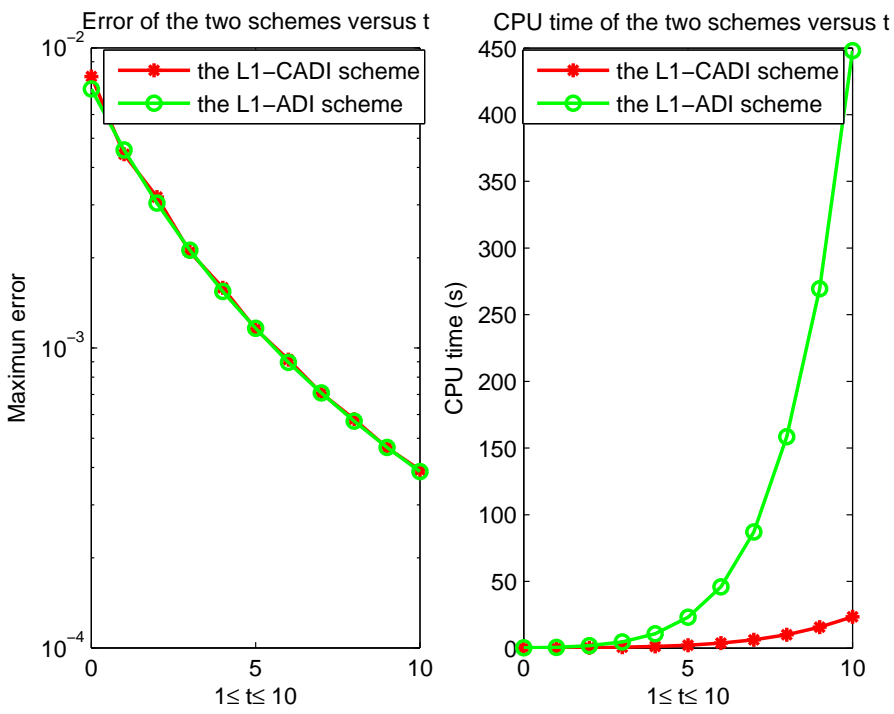

Figure 2: Error and CPU time of the L1-CADI and L1-ADI schemes.

approximation is derived that has fourth-order spatial accuracy $[19,21,33]$. Using some novel techniques, the unique solvability, unconditional stability and global convergence are proved rigorously for both the $1 \mathrm{D}$ and $2 \mathrm{D}$ cases. Numerical examples verify the effectiveness of the compact difference scheme. These methods and techniques could be extended to other kinds of multi-term time-space fractional equations and to equations with a nonlinear source term. Future work may concentrate on studying the effective numerical schemes for the multi-term fractional time-space equations. 


\section{Acknowledgments}

We express our gratitude to the editor and referees for valuable comments and suggestions. This research is supported by the National Natural Science Foundation of China (No. 11271068), the Foundation of Henan Educational Committee (No. 13B110190) and the Natural Science Foundation of Henan Province (No. 142300410115).

\section{References}

[1] I. Podlubny, Fractional Differential Equations, Academic Press, New York, 1999.

[2] M.M. Meerschaert, D. Benson and B. Baeumer, Operator Lévy motion and multiscaling anomalous diffusion, Phys. Rev. E 63, 1112-1117 (2001).

[3] T.H. Solomon, E.R. Weeks and H.L. Swinney, Observations of anomalous diffusion and Léry flights in a 2-dimensional rotating flow, Phys. Rev. Lett. 71, 3975-3979 (1993).

[4] S.B. Yuste and K. Lindenberg, Subdiffusion-limited A+A reactions, Phys. Rev. Lett. 87, 118301 (2001).

[5] M. Raberto, E. Scalas and F. Mainardi, Waiting-times and returns in high-frequency financial data: an empirical study, Phys. A 314, 749-755 (2002).

[6] F. Mainardi, M. Raberto, R. Gorenflo and E. Scalas, Fractional calculus and continuous-time finance II: the waiting-time distribution, Phys. A 287, 468-481 (2000).

[7] D.A. Benson, S.W. Wheatcraft and M.M. Meerschaert, Application of a fractional advectiondispersion equation, Water Resources Res. 36, 1403-1412 (2000).

[8] S. Vong and Z.B. Wang, High order difference schemes for a time fractional differential equation with Neumann boundary conditions, East Asian J. Appl. Math. 4 (to appear).

[9] R.L. Bagley and P.J. Torvik, On the appearance of the fractional derivative in the behaviour of real materials, J. Appl. Mech. 51, 294-298 (1984).

[10] Y. Luchko, Initial-boundary-value problems for the generalized multi-term time fractional diffusion equation, J. Math. Anal. Appl. 374, 538-548 (2011).

[11] V. Daftardar-Gejji and S. Bhalekar, Boundary value problems for multi-term fractional differential equations, J. Math. Anal. Appl. 345, 754-765 (2008).

[12] H. Jiang, F. Liu, I. Turner and K. Burrage, Analytical solutions for the multi-term time-space Caputo-Riesz fractional advection-diffusion equations on a finite domain, J. Math. Anal. Appl. 389, 1117-1127 (2012).

[13] H. Jiang, F. Liu, M.M. Meerschaert and R. McGough, Fundamental solutions for the multi-term modified power law wave equations in a finite domain, Elec. J. Math. Anal. Appl. 1, 55-66 (2013).

[14] C. Chen, F. Liu, I. Turner and V. Anh, A Fourier method for the fractional diffusion equation describing sub-diffusion, J. Comput. Phys. 227, 886-897 (2007).

[15] V.E. Lynch, B.A. Carreras and D. Del-Castill-Negrete, Numerical methods for the solution of partial differential equations of fractional order, J. Comput. Phys. 192, 406-421 (2003).

[16] S.B. Yuste and L. Acedo, An explicit finite difference method and a new von Neumann-type stability analysis for fractional diffusion equations, SIAM J. Numer. Anal. 42, 1862-1874 (2005).

[17] S.B. Yuste, Weighted average finite difference metods for fractional diffusion equations, J. Comput. Phys. 216, 264-274 (2006).

[18] P. Zhuang, F. Liu, V. Anh and I. Turner, New solution and analytical techniques of the implicit numerical method for the anomalous subdiffusion equation, SIAM J. Numer. Anal. 46, 10791095 (2008). 
[19] Y.N. Zhang, Z.Z. Sun and H.W. Wu, Error estimates of Crank-Nicolson-type difference scheme for the subdiffusion equation, SIAM J. Numer. Anal. 49, 2302-2322 (2011).

[20] X. Zhao and Z.Z. Sun, A box-type scheme for the fractonal sub-diffusion equation with Neumann boundary conditions, J. Comput. Phys. 230, 6061-6074 (2011).

[21] J.C. Ren, Z.Z. Sun and X. Zhao, Compact difference scheme for the fractional sub-diffusion equation with Neumann boundary conditions, J. Comput. Phys. 232, 456-467 (2013).

[22] C.P. Li, Z.G. Zhao and Y.Q. Chen, Numerical approximation of nonlinear fractional differential equations with subdiffusion and superdiffusion, Comput. Math. Appl. 62, 855-875 (2011).

[23] W.H. Deng, Numerical algorithm for the time fractional Fokker-Planck equation, J. Comput. Phys. 227, 1510-1522 (2007).

[24] V.J. Ervin, N. Heuer and J.P. Roop, Numerical approximation of a time dependent, nonlinear, space-fractional diffusion equation, SIAM J. Numer. Anal. 45, 572-591 (2007).

[25] N.J. Ford, J.Y. Xiao and Y.B. Yan, Stability of a numerical method for a space-time fractional telegraph equation, Comput. Meth. Appl. Math. 12, 1-16 (2012).

[26] F. Liu, C. Yang and K. Burrage, Numerical method and analytical technique of the modifed anomalous subdiffusion equation with a nonlinear source term, J. Comput. Appl. Math., 231, 160-176 (2009).

[27] Q. Liu, F. Liu, I. Turner and V. Anh, Finite element approximation for a modifed anomalous subdiffusion equation, Appl. Math. Model. 35, 4103-4116 (2011).

[28] F. Liu, M.M. Meerschaert, R. McGough, P. Zhuang and Q. Liu, Numerical methods for solving the multi-term time fractional wave equations, Fractional Calculus \& Applied Analysis, 16, 9-25 (2013).

[29] S.P. Yang, A.G. Xiao and H. Su, Convergence of the variational iteration method for sovling multi-order fractional differential equations, Comput. Math. Appl. 60, 2871-2879 (2010).

[30] Y. Lin and C.J. Xu, Finite difference/spectral approximations for the time fractional diffusion equation, J. Comput. Phys. 225, 1533-1552 (2007).

[31] Z.Z. Sun and X.N. Wu, A fully discrete difference scheme for a diffusion-wave system, Appl. Numer. Math. 56, 193-209 (2006).

[32] Z.Z. Sun, Compact difference schemes for heat equation with Neumann boundary conditions, Numer. Methods Partial Differential Equations 25, 1320-1341 (2009).

[33] J.C. Ren and Z.Z. Sun, Numerical algorithm with high spatial accuracy for the fractional diffusion-wave equation with Neumann boundary conditions, J. Sci. Comput. 56, 381-408 (2013).

[34] Z.Z. Sun, Numerical Methods of Partial Differential Equations (2nd edition), Science Press, Beijing, 2012.

[35] S.D. Conte and C. de Boor, Elementary Numerical Analysis: An Algorithmic Approach (3rd edition), McGraw-Hill, New York, 1980. 